\title{
Deligne-Beilinson Cohomology and Abelian Link Invariants
}

\author{
Enore GUADAGNINI ${ }^{\dagger}$ and Frank THUILLIER ${ }^{\ddagger}$ \\ $\dagger$ Dipartimento di Fisica "E. Fermi" dell'Università di Pisa \\ and Sezione di Pisa dell'INFN, Italy \\ E-mail: enore.guadagnini@df.unipi.it \\ URL: http://www.df .unipi.it/ guada/ \\ $\ddagger$ LAPTH, Chemin de Bellevue, BP 110, F-74941 Annecy-le-Vieux cedex, France \\ E-mail: frank.thuilier@lapp.in2p3.fr \\ URL: http://lappweb.in2p3.fr/ thuillie/
}

Received July 14, 2008, in final form October 27, 2008; Published online November 11, 2008

Original article is available at http://www.emis.de/journals/SIGMA/2008/078/

\begin{abstract}
For the Abelian Chern-Simons field theory, we consider the quantum functional integration over the Deligne-Beilinson cohomology classes and we derive the main properties of the observables in a generic closed orientable 3-manifold. We present an explicit pathintegral non-perturbative computation of the Chern-Simons link invariants in the case of the torsion-free 3-manifolds $S^{3}, S^{1} \times S^{2}$ and $S^{1} \times \Sigma_{g}$.

Key words: Deligne-Beilinson cohomology; Abelian Chern-Simons; Abelian link invariants
\end{abstract}

2000 Mathematics Subject Classification: 81T70; 14F43; 57M27

\section{Introduction}

The topological quantum field theory which is defined by the Chern-Simons action can be used to compute invariants of links in 3-manifolds [1, 2, 3, 4]. The algebraic structure of these invariants, which is based on the properties of the characters of simple Lie groups, is rather general. In fact, these invariants can also be defined by means of skein relations or of quantum group Hopf algebra methods [5, 6].

In the standard quantum field theory approach, the gauge invariance group of the Abelian Chern-Simons theory is given by the set of local $U(1)$ gauge transformations and the observables can directly be computed by means of perturbation theory when the ambient space is $\mathbb{R}^{3}$ (the result also provides the values of the link invariants in $S^{3}$ ). For a nontrivial 3-manifold $M_{3}$, the standard gauge theory approach presents some technical difficulties, and one open problem of the quantum Chern-Simons theory is to produce directly the functional integration in the case of a generic 3-manifold $M_{3}$. In this article we will show how this can be done, at least for a certain class of nontrivial 3-manifolds, by using the Deligne-Beilinson cohomology. We shall concentrate on the Abelian Chern-Simons invariants; hopefully, the method that we present will possibly admit an extension to the non-Abelian case.

The Deligne-Beilinson approach presents some remarkable aspects. The space of classical field configurations which are factorized out by gauge invariance is enlarged with respect to the standard field theory formalism. Indeed, assuming that the quantum amplitudes given by the exponential of the holonomies - which are associated with oriented loops - represent a complete set of observables, the functional integration must locally correspond to a sum over 1forms modulo forms with integer periods, i.e. it must correspond to a sum over Deligne-Beilinson classes. In this new approach, the structure of the functional space admits a natural description 
in terms of the homology groups of the 3-manifold $M_{3}$. This structure will be used to compute the Chern-Simons observables, without the use of perturbation theory, on a class of torsion-free manifolds.

The article is organized as follows. Section 2 contains a description of the basic properties of the Deligne-Beilinson cohomology and of the distributional extension of the space of the equivalence classes. The framing procedure is introduced in Section 3. The general properties of the Abelian Chern-Simons theory are discussed in Section 4; in particular, non-perturbative proofs of the colour periodicity, of the ambient isotopy invariance and of the satellite relations are given. The solution of the Chern-Simons theory on $S^{3}$ is presented in Section 5. The computations of the observables for the manifolds $S^{1} \times S^{2}$ and $S^{1} \times \Sigma_{g}$ are produced in Sections 6 and 7 . Section 8 contains a brief description of the surgery rules that can be used to derive the link invariants in a generic 3-manifold, and it is checked that the results obtained by means of the Deligne-Beilinson cohomology and by means of the surgery method coincide. Finally, Section 9 contains the conclusions.

\section{Deligne-Beilinson cohomology}

The applications of the Deligne-Beilinson (DB) cohomolgy [7, 8, 9, 10, 11] - and of its various equivalent versions such as the Cheeger-Simons Differential Characters [12, 13] or Sparks [14] in quantum physics has been discussed by various authors $[15,16,17,18,19,21,20,22$, 23]. For instance, geometric quantization is based on classes of $U(1)$-bundles with connections, which are exactly DB classes of degree one (see Section 8.3 of [24]); and the Aharanov-Bohm effect also admits a natural description in terms of DB cohomology.

In this article, we shall consider the computation of the Abelian link invariants of the ChernSimons theory by means of the DB cohomology. Let $L$ be an oriented (framed and coloured) link in the 3-manifold $M_{3}$; one is interested in the ambient isotopy invariant which is defined by the path-integral expectation value

$$
\left\langle\exp \left\{2 i \pi \int_{L} A\right\}\right\rangle_{k} \equiv \frac{\int D A \exp \left\{2 i \pi k \int_{M_{3}} A \wedge d A\right\} \exp \left\{2 i \pi \int_{L} A\right\}}{\int D A \exp \left\{2 i \pi k \int_{M_{3}} A \wedge d A\right\}}
$$

where the parameter $k$ represents the dimensionless coupling constant of the field theory. In equation (2.1), the holonomy associated with the link $L$ is defined in terms of a $U(1)$-connection $A$ on $M_{3}$; this holonomy is closely related to the classes of $U(1)$-bundles with connections that represent DB cohomology classes. The Chern-Simons lagrangian $A \wedge d A$ can be understood as a DB cohomology class from the Cheeger-Simons Differential Characters point of view, and it can also be interpreted as a DB "square" of $A$ which is defined, as we shall see, by means of the DB $*$-product.

To sum up, the DB cohomology appears to be the natural framework which should be used in order to compute the Chern-Simons expectation values (2.1). As we shall see, this will imply the quantization of the coupling constant $k$ and it will actually provide the integration measure $D A$ with a nontrivial structure which is related to the homology of the manifold $M_{3}$. It should be noted that the gauge invariance of the Chern-Simons action and of the observables is totally included into the DB setting: working with DB classes means that we have already taken the quotient by gauge transformations.

Although we won't describe DB cohomology in full details, we shall now present a few properties of the DB cohomology that will be useful for the non-perturbative computation of the observables (2.1). 


\subsection{General properties}

Let $M$ be a smooth oriented compact manifold without boundary of finite dimension $n$. The Deligne cohomology group of $M$ of degree $q, H_{D}^{q}(M, \mathbb{Z})$, can be described as the central term of the following exact sequence

$$
0 \longrightarrow \Omega^{q}(M) / \Omega_{\mathbb{Z}}^{q}(M) \longrightarrow H_{D}^{q}(M, \mathbb{Z}) \longrightarrow H^{q+1}(M, \mathbb{Z}) \longrightarrow 0,
$$

where $\Omega^{q}(M)$ is the space of smooth $q$-forms on $M, \Omega_{\mathbb{Z}}^{q}(M)$ the space of smooth closed $q$-forms with integral periods on $M$ and $H^{q+1}(M, \mathbb{Z})$ is the $(q+1)^{t h}$ integral cohomology group of $M$. This last space can be taken as simplicial, singular or Cech. There is another exact sequence into which $H_{D}^{q}(M, \mathbb{Z})$ can be embedded, namely

$$
0 \longrightarrow H^{q}(M, \mathbb{R} / \mathbb{Z}) \longrightarrow H_{D}^{q}(M, \mathbb{Z}) \longrightarrow \Omega_{\mathbb{Z}}^{q+1}(M) \longrightarrow 0,
$$

where $H^{q}(M, \mathbb{R} / \mathbb{Z})$ is the $\mathbb{R} / \mathbb{Z}$-cohomology group of $M[11,14,25]$.

One can compute $H_{D}^{q}(M, \mathbb{Z})$ by using a (hyper) cohomological resolution of a double complex of Cech-de Rham type, as explained for instance in $[9,25]$. In this approach, $H_{D}^{q}(M, \mathbb{Z})$ appears as the set of equivalence classes of DB cocycles which are defined by sequences $\left(\omega^{(0, q)}, \omega^{(1, q-1)}\right.$, $\left.\ldots, \omega^{(q, 0)}, \omega^{(q+1,-1)}\right)$, where $\omega^{(p, q-p)}$ denotes a collection of smooth $(q-p)$-forms in the intersections of degree $p$ of some open sets of a good open covering of $M$, and $\omega^{(q+1,-1)}$ is an integer Cech $(p+1)$-cocyle for this open good covering of $M$. These forms satisfy cohomological descent equations of the type $\delta \omega^{(p-1, q-p+1)}+d \omega^{(p, q-p)}=0$, and the equivalence relation is defined via the $\delta$ and $d$ operations, which are respectively the Cech and de Rham differentials. The Cechde Rham point of view has the advantage of producing "explicit" expressions for representatives of DB classes in some good open covering of $M$.

Definition 2.1. Let $\omega$ be a $q$-form which is globally defined on the manifold $M$. We shall denote by $[\omega] \in H_{D}^{q}(M, \mathbb{Z})$ the DB class which, in the Cech-de Rham double complex approach, is represented by the sequence $\left(\omega^{(0, q)}=\omega, \omega^{(1, q-1)}=0, \ldots, \omega^{(q, 0)}=0, \omega^{(q+1,-1)}=0\right)$.

From sequence $(2.2)$ it follows that $H_{D}^{q}(M, \mathbb{Z})$ can be understood as an affine bundle over $H^{q+1}(M, \mathbb{Z})$, whose fibres have a typical underlying (infinite dimensional) vector space structure given by $\Omega^{q}(M) / \Omega_{\mathbb{Z}}^{q}(M)$. Equivalently, $\Omega^{q}(M) / \Omega_{\mathbb{Z}}^{q}(M)$ canonically acts on the fibres of the bundle $H_{D}^{q}(M, \mathbb{Z})$ by translation. From a geometrical point of view, $H_{D}^{1}(M, \mathbb{Z})$ is canonically isomorphic to the space of equivalence classes of $U(1)$-principal bundles with connections over $M$ (see for instance [14, 25]). A generalisation of this idea has been proposed by means of Abelian Gerbes (see for instance [11, 26]) and Abelian Gerbes with connections over $M$. In this framework, $H^{q+1}(M, \mathbb{Z})$ classifies equivalence classes of some Abelian Gerbes over $M$, in the same way as $H^{2}(M, \mathbb{Z})$ is the space which classifies the $U(1)$-principal bundles over $M$, and $H_{D}^{q}(M, \mathbb{Z})$ appears as the set of equivalence classes of some Abelian Gerbes with connections. Finally, the space $\Omega_{\mathbb{Z}}^{q}(M)$ can be interpreted as the group of generalised Abelian gauge transformations.

We shall mostly be concerned with the cases $q=1$ and $q=3$. As for $M$, we will consider the three dimensional cases $M_{3}=S^{3}, M_{3}=S^{1} \times S^{2}$ and $M_{3}=S^{1} \times \Sigma_{g}$, where $\Sigma_{g}$ is a Riemann surface of genus $g \geq 1$. In particular, $M_{3}$ is oriented and torsion free. In all these cases, the exact sequence $(2.2)$ for $q=3$ reads

$$
0 \longrightarrow \Omega^{3}\left(M_{3}\right) / \Omega_{\mathbb{Z}}^{3}\left(M_{3}\right) \longrightarrow H_{D}^{3}\left(M_{3}, \mathbb{Z}\right) \longrightarrow H^{4}\left(M_{3}, \mathbb{Z}\right)=0 \longrightarrow 0,
$$

where the first non trivial term reduces to

$$
\frac{\Omega^{3}\left(M_{3}\right)}{\Omega_{\mathbb{Z}}^{3}\left(M_{3}\right)} \cong \frac{\mathbb{R}}{\mathbb{Z}} .
$$




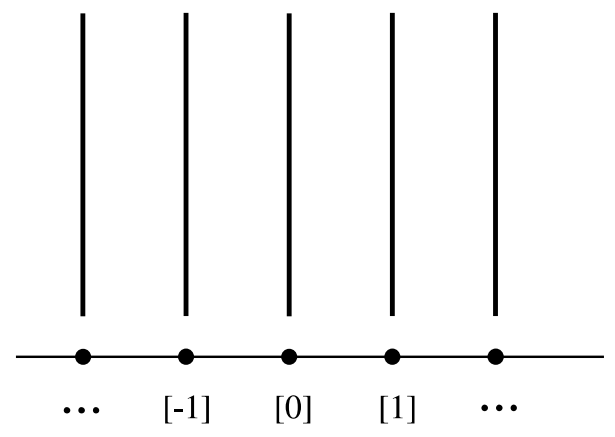

Figure 1. Presentation of the Deligne-Beilinson affine bundle $H_{D}^{1}\left(S^{1} \times S^{2}, \mathbb{Z}\right)$.

The validity of equation (2.4) can easily be checked by using a volume form on $M_{3}$. By definition, for any $\left(\rho, \tau_{\mathbb{Z}}\right) \in \Omega^{3}\left(M_{3}\right) \times \Omega_{\mathbb{Z}}^{3}\left(M_{3}\right)$ one has

$$
\left[\rho+\tau_{\mathbb{Z}}\right]=[\rho] \in H_{D}^{3}\left(M_{3}, \mathbb{Z}\right) ;
$$

consequently

$$
H_{D}^{3}\left(M_{3}, \mathbb{Z}\right) \simeq \frac{\Omega^{3}\left(M_{3}\right)}{\Omega_{\mathbb{Z}}^{3}\left(M_{3}\right)} \cong \frac{\mathbb{R}}{\mathbb{Z}}
$$

These results imply that any Abelian 2-Gerbes on $M_{3}$ is trivial $\left(H^{4}\left(M_{3}, \mathbb{Z}\right)=0\right)$, and the set of classes of Abelian 2-Gerbes with connections on $M_{3}$ is isomorphic to $\mathbb{R} / \mathbb{Z}$. In the less trivial case $q=1$, sequence $(2.2)$ reads

$$
0 \longrightarrow \Omega^{1}\left(M_{3}\right) / \Omega_{\mathbb{Z}}^{1}\left(M_{3}\right) \longrightarrow H_{D}^{1}\left(M_{3}, \mathbb{Z}\right) \longrightarrow H^{2}\left(M_{3}, \mathbb{Z}\right) \longrightarrow 0 .
$$

Still by definition, for any $\left(\eta, \omega_{\mathbb{Z}}\right) \in \Omega^{1}\left(M_{3}\right) \times \Omega_{\mathbb{Z}}^{1}\left(M_{3}\right)$ one has

$$
\left[\eta+\omega_{\mathbb{Z}}\right]=[\eta] \in H_{D}^{1}\left(M_{3}, \mathbb{Z}\right) .
$$

When $H^{2}\left(M_{3}, \mathbb{Z}\right)=0$, sequence (2.5) turns into a short exact sequence; this also implies $H^{1}\left(M_{3}, \mathbb{Z}\right)=0$ due to Poincaré duality. For the 3 -sphere $S^{3}$, the base space of $H_{D}^{1}\left(S^{3}, \mathbb{Z}\right)$ is trivial. Whereas, the bundle $H_{D}^{1}\left(S^{1} \times S^{2}, \mathbb{Z}\right)$ has base space $H^{2}\left(S^{1} \times S^{2}, \mathbb{Z}\right) \cong \mathbb{Z}$ and, as depicted in Fig. 1, its fibres are (infinite dimensional) affine spaces whose underlying linear space identifies with the quotient space $\Omega^{1}\left(S^{1} \times S^{2}\right) / \Omega_{\mathbb{Z}}^{1}\left(S^{1} \times S^{2}\right)$. In the general case $M_{3}=S^{1} \times \Sigma_{g}$ with $g \geq 1$, the base space $H^{2}\left(S^{1} \times \Sigma_{g}, \mathbb{Z}\right)$ is isomorphic to $\mathbb{Z}^{2 g+1}$.

Finally, one should note that sequence (2.5) also gives information on $\Omega_{\mathbb{Z}}^{1}\left(M_{3}\right)$ since its structure is mainly given by the $H_{D}^{1}\left(M_{3}, \mathbb{Z}\right)$. For instance, $\Omega_{\mathbb{Z}}^{1}\left(S^{3}\right)=d \Omega^{0}\left(S^{3}\right)$, all other cases being not so trivial.

\subsection{Holonomy and pairing}

As we have already mentioned, DB cohomology is the natural framework in which integration (or holonomy) of a $U$ (1)-connection over 1-cycles of $M_{3}$ can be defined and generalised to objects of higher dimension ( $n$-connections and $n$-cycles). In fact integration of a DB cohomology class $[\chi] \in H_{D}^{q}(M, \mathbb{Z})$ over a $q$-cycle of $M$, denoted by $C \in Z_{q}(M)$, appears as a $\mathbb{R} / \mathbb{Z}$-valued linear pairing

$$
\begin{aligned}
\langle,\rangle_{q}: & H_{D}^{q}(M, \mathbb{Z}) \times Z_{q}(M) \longrightarrow \mathbb{R} / \mathbb{Z}=S^{1}, \\
& ([\chi], C) \longrightarrow\langle[\chi], C\rangle_{q} \equiv \int_{C}[\chi],
\end{aligned}
$$


which establishes the equivalence between DB cohomology and Cheeger-Simons characters [12, 13, 11, 14, 25]. Accordingly, a quantity such as

$$
\exp \left\{2 i \pi \int_{C}[\chi]\right\}
$$

is well defined and corresponds to the fundamental representation of $\mathbb{R} / \mathbb{Z}=S^{1} \simeq U(1)$. Using the Chech-de Rham description of DB cocycles, one can then produce explicit formulae [25] for the pairing (2.6).

Alternatively, (2.6) can be seen as a dualising equation. More precisely, any $C \in Z_{q}(M)$ belongs to the Pontriagin dual of $H_{D}^{q}(M, \mathbb{Z})$, usually denoted by $\operatorname{Hom}\left(H_{D}^{q}(M, \mathbb{Z}), S^{1}\right)$, the pairing (2.6) providing a canonical injection

$$
Z_{q}(M) \vec{\subset} \operatorname{Hom}\left(H_{D}^{q}(M, \mathbb{Z}), S^{1}\right) .
$$

A universal result [27] about the Hom functor implies the validity of the exact sequences, dualising (2.2) (via (2.3)),

$$
0 \longrightarrow \operatorname{Hom}\left(\Omega_{\mathbb{Z}}^{q+1}(M), S^{1}\right) \longrightarrow \operatorname{Hom}\left(H_{D}^{q}(M, \mathbb{Z}), S^{1}\right) \longrightarrow H^{n-q}(M, \mathbb{Z}) \longrightarrow 0,
$$

where $H^{n-q}(M, \mathbb{Z}) \cong \operatorname{Hom}\left(H^{q}(M, \mathbb{R} / \mathbb{Z}), S^{1}\right)$.

The space $\operatorname{Hom}\left(H_{D}^{q}(M, \mathbb{Z}), S^{1}\right)$ also contains $H_{D}^{n-q-1}(M, \mathbb{Z})$, so that $Z_{q}(M)$ (or rather its canonical injection $(2.7)$ ) can be seen as lying on the boundary of $H_{D}^{n-q-1}(M, \mathbb{Z}$ ) (see details in [14]). Accordingly

$$
Z_{q}(M) \oplus H_{D}^{n-q-1}(M, \mathbb{Z}) \subset \operatorname{Hom}\left(H_{D}^{q}(M, \mathbb{Z}), S^{1}\right),
$$

with the obvious abuse in the notation. Let us point out that, as suggested by equation (2.9), one could represent integral cycles by currents which are singular (i.e. distributional) forms. This issue will be discussed in detail in next subsection.

Now, sequence $(2.8)$ shows that $\operatorname{Hom}\left(H_{D}^{q}(M, \mathbb{Z}), S^{1}\right)$ is also an affine bundle with base space $H^{n-q}(M, \mathbb{Z})$. In particular, let us consider the case in which $n=3$ and $q=1$; on the one hand, Poincaré duality implies

$$
H^{n-q}(M, \mathbb{Z})=H^{2}\left(M_{3}, \mathbb{Z}\right) \cong H^{1}\left(M_{3}, \mathbb{Z}\right) .
$$

On the other hand, one has

$$
H_{D}^{1}(M, \mathbb{Z}) \subset \operatorname{Hom}\left(H_{D}^{1}(M, \mathbb{Z}), S^{1}\right),
$$

and, because of the Pontriagin duality,

$$
Z_{1}(M) \oplus H_{D}^{1}(M, \mathbb{Z}) \subset \operatorname{Hom}\left(H_{D}^{1}(M, \mathbb{Z}), S^{1}\right) .
$$

This is somehow reminiscent of the self-dual situation in the case of four dimensional manifolds and curvature.

\subsection{The product}

The pairing (2.6) is actually related to another pairing of DB cohomology groups

$$
H_{D}^{p}(M, \mathbb{Z}) \times H_{D}^{q}(M, \mathbb{Z}) \longrightarrow H_{D}^{p+q+1}(M, \mathbb{Z}),
$$

whose explicit description can be found for instance in $[12,14,25]$. This pairing is known as the DB product (or DB *-product). It will be denoted by *. In the Cech-de Rham approach, 
the DB product of the DB cocyle $\left(\omega^{(0, p)}, \omega^{(1, p-1)}, \ldots, \omega^{(p, 0)}, \omega^{(p+1,-1)}\right)$ with the DB cocycle $\left(\eta^{(0, q)}, \eta^{(1, q-1)}, \ldots, \eta^{(q, 0)}, \eta^{(q+1,-1)}\right)$ reads

$$
\left(\omega^{(0, p)} \cup d \eta^{(0, q)}, \ldots, \omega^{(p, 0)} \cup d \eta^{(0, q)}, b \omega^{(p+1,-1)} \cup \eta^{(0, q)}, \ldots, \omega^{(p+1,-1)} \cup \eta^{(n-p,-1)}\right),
$$

where the product $\cup$ is precisely defined in $[28,9,25]$, for instance.

Definition 2.2. Let us consider the sequence $\left(\eta^{(0, q)}, \eta^{(1, q-1)}, \ldots, \eta^{(q, 0)}, \eta^{(q+1,-1)}\right)$, in which the components $\eta^{(k-q, k)}$ satisfy the same descent equations as the components of a DB cocycle but, instead of smooth forms, these components are currents (i.e. distributional forms). This allows to extend the (smooth) cohomology group $H_{D}^{q}(M, \mathbb{Z})$ to a larger cohomology group that we will denote $\widetilde{H}_{D}^{q}(M, \mathbb{Z})$.

Obviously, the DB product (2.11) of a smooth DB cocycle with a distributional one is still well-defined, and thus the pairing (2.10) extends to

$$
H_{D}^{p}(M, \mathbb{Z}) \times \widetilde{H}_{D}^{q}(M, \mathbb{Z}) \longrightarrow \widetilde{H}_{D}^{p+q+1}(M, \mathbb{Z}) .
$$

Then, it can be checked [25] that any class $[\eta] \in \widetilde{H}_{D}^{n-q-1}(M, \mathbb{Z})$ canonically defines a $\mathbb{R} / \mathbb{Z}$-valued linear pairing as in (2.6) so that

$$
\widetilde{H}_{D}^{n-q-1}(M, \mathbb{Z}) \subset \operatorname{Hom}\left(H_{D}^{q}(M, \mathbb{Z}), S^{1}\right) .
$$

It is important to note that, as it was shown in [25], to any $C \in Z_{q}(M)$ there corresponds a canonical DB class $\left[\eta_{C}\right] \in \widetilde{H}_{D}^{n-q-1}(M, \mathbb{Z})$ such that

$$
\exp \left\{2 i \pi \int_{C}[\chi]\right\}=\exp \left\{2 i \pi \int_{M}[\chi] *\left[\eta_{C}\right]\right\}
$$

for any $[\chi] \in H_{D}^{q}(M, \mathbb{Z})$. This means that we have the following sequence of canonical inclusions

$$
Z_{q}(M) \subset \widetilde{H}_{D}^{n-q-1}(M, \mathbb{Z}) \subset \operatorname{Hom}\left(H_{D}^{q}(M, \mathbb{Z}), S^{1}\right) .
$$

Let us point out the trivial inclusion

$$
H_{D}^{n-q-1}(M, \mathbb{Z}) \subset \widetilde{H}_{D}^{n-q-1}(M, \mathbb{Z}) .
$$

In the 3 dimensional case, let us consider the $\mathrm{DB}$ product

$$
H_{D}^{1}\left(M_{3}, \mathbb{Z}\right) \times H_{D}^{1}\left(M_{3}, \mathbb{Z}\right) \longrightarrow H_{D}^{3}\left(M_{3}, \mathbb{Z}\right) \cong \mathbb{R} / \mathbb{Z}
$$

Starting from equation (2.12) and extending it to

$$
H_{D}^{1}\left(M_{3}, \mathbb{Z}\right) \times \widetilde{H}_{D}^{1}\left(M_{3}, \mathbb{Z}\right) \longrightarrow \widetilde{H}_{D}^{3}\left(M_{3}, \mathbb{Z}\right) \cong \mathbb{R} / \mathbb{Z},
$$

one finds that it is possible to associate with any 1-cycle $C \in Z_{1}\left(M_{3}\right)$ a canonical DB class $\left[\eta_{C}\right] \in \widetilde{H}_{D}^{1}\left(M_{3}, \mathbb{Z}\right)$ such that

$$
\exp \left\{2 i \pi \int_{C}[\omega]\right\}=\exp \left\{2 i \pi \int_{M_{3}}[\omega] *\left[\eta_{C}\right]\right\}
$$

for any $[\omega] \in H_{D}^{1}\left(M_{3}, \mathbb{Z}\right)$. As an an alternative point of view, consider a smoothing homotopy of $C$ within $H_{D}^{1}\left(M_{3}, \mathbb{Z}\right)$, that is, a sequence of smooth DB classes $\left[\eta_{\varepsilon}\right] \in H_{D}^{1}(M, \mathbb{Z})$ such that (see [14] for details)

$$
\lim _{\varepsilon \rightarrow 0} \exp \left\{2 i \pi \int_{M}[A] *\left[\eta_{\varepsilon}\right]\right\}=\exp \left\{2 i \pi \int_{C}[A]\right\} \text {. }
$$




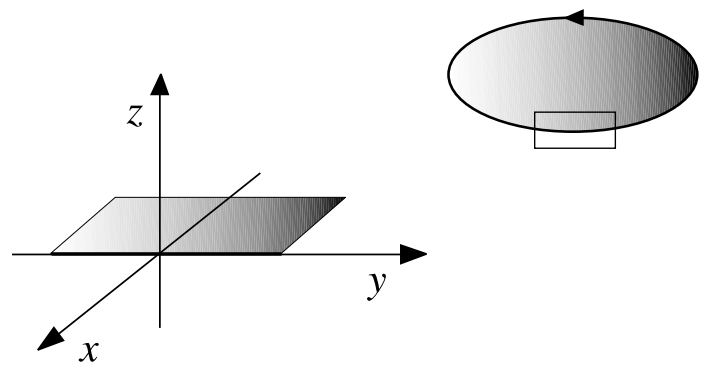

Figure 2. In a open domain with local coordinates $(x, y, z)$, a piece of a homologically trivial loop $C$ can be identified with the $y$ axis, and the disc that it bounds (Seifert surface) can be identified with a portion of the half plane $(x<0, y, z=0)$.

This implies

$$
\lim _{\varepsilon \rightarrow 0}\left[\eta_{\varepsilon}\right]=\left[\eta_{C}\right]
$$

within the completion $\widetilde{H}_{D}^{1}\left(M_{3}, \mathbb{Z}\right)$ of $H_{D}^{1}\left(M_{3}, \mathbb{Z}\right)$; this is why in [14] [ $\left.\eta_{C}\right]$ is said to belong to the boundary of $H_{D}^{1}\left(M_{3}, \mathbb{Z}\right)$. It should be noted that, by definition, the limit (2.14) and the corresponding limit (2.15) are always well defined. For this reason, in what follows we shall concentrate directly to the distributional space $\widetilde{H}_{D}^{1}\left(M_{3}, \mathbb{Z}\right)$ and, in the presentation of the various arguments, the possibility of adopting a limiting procedure of the type shown in equation (2.14) will be simply understood.

Finally, let us point out that with the aforementioned geometrical interpretation of DB cohomology classes, the DB product of smooth classes canonically defines a product within the space of Abelian Gerbes with connections. For instance, the DB product of two classes of $U(1)$-bundles with connections over $M$ turns out to be a class of $U(1)$-gerbe with connection over $M$.

\subsection{Distributional forms and Seifert surfaces}

How to construct the class $\left[\eta_{C}\right]$, which enters equation (2.13), is explained in detail for instance in [25]. Here we outline the main steps of the construction and we consider, for illustrative purposes, the case $M_{3} \sim S^{3}$. The integral of a one-form $\omega$ along an oriented knot $C \subset S^{3}$ can be written as the integral on the whole $S^{3}$ of the external product $\omega \wedge J_{C}$, where the current $J_{C}$ is a distributional 2-form with support on the $\operatorname{knot} C$; that is, $\int_{C} \omega=\int_{S^{3}} \omega \wedge J_{C}$. Since $J_{C}$ can be understood as the boundary of an oriented surface $\Sigma_{C}$ in $S^{3}$ (called a Siefert surface), one has $J_{C}=d \eta_{C}$ for some 1 -form $\eta_{C}$ with support on $\Sigma_{C}$. One then finds, $\int_{C} \omega=\int_{S^{3}} \omega \wedge d \eta_{C}$, which corresponds precisely to equation $(2.13)$ with $\left[\eta_{C}\right] \in \widetilde{H}_{D}^{1}\left(S^{3}, \mathbb{Z}\right)$ denoting the Deligne cohomology class which is associated to $\eta_{C}$ and with $[\omega] \in H_{D}^{1}\left(S^{3}, \mathbb{Z}\right)$ denoting the class which can be represented by $\omega$.

Let us consider, for instance, the unknot $C$ in $S^{3}$, shown in Fig. 2, with a simple disc as Seifert surface. Inside the open domain depicted in Fig. 2, the oriented knot is described - in local coordinates $(x, y, z)$ - by a piece of the $y$-axis and the corresponding distributional forms $J_{C}$ and $\eta_{C}$ are given by

$$
J_{C}=\delta(z) \delta(x) d z \wedge d x, \quad \eta_{C}=\delta(z) \theta(-x) d z .
$$

For a generic 3-manifold $M_{3}$ and for each oriented knot $C \subset M_{3}$, the distributional 2form $J_{C}$ always exists, whereas a corresponding Seifert surface and the associated 1-form $\eta_{C}$ can in general be (globally) defined only when the second cohomology group of $M_{3}$ is vanishing. Nevertheless, the class $\left[\eta_{C}\right] \in \widetilde{H}_{D}^{1}(M, \mathbb{Z})$ is always well defined for arbitrary 3-manifold $M_{3}$. In 
fact, when a Seifert surface associated with $C \subset M_{3}$ does not exist, the Chech-de Rham cocycle sequence representing $\left[\eta_{C}\right] \in \widetilde{H}_{D}^{1}(M, \mathbb{Z})$ is locally of the form $\left(\eta_{C}^{(0,1)}, \Lambda_{C}^{(1,0)}, N_{C}^{(2,-1)}\right)$ where, inside sufficiently small open domains, the expression of $\eta_{C}^{(0,1)}$ is trivial or may coincide with the expression (2.16) for $\eta_{C}$, and $\Lambda_{C}^{(1,0)}$ and $N_{C}^{(2,-1)}$ are nontrivial components (when a Seifert surface exists, the components $\Lambda_{C}^{(1,0)}$ and $N_{C}^{(2,-1)}$ are trivial).

\section{Linking and self-linking}

As we have already mentioned, in the context of equation (2.13) the pairing $H_{D}^{1}\left(M_{3}, \mathbb{Z}\right) \times$ $\widetilde{H}_{D}^{1}\left(M_{3}, \mathbb{Z}\right) \rightarrow \widetilde{H}_{D}^{3}\left(M_{3}, \mathbb{Z}\right)$ is well defined. However, in what follows we shall also need to consider a pairing induced by the DB product of the type $\widetilde{H}_{D}^{1}\left(M_{3}, \mathbb{Z}\right) \times \widetilde{H}_{D}^{1}\left(M_{3}, \mathbb{Z}\right) \rightarrow \widetilde{H}_{D}^{3}\left(M_{3}, \mathbb{Z}\right)$ and this presents in general ambiguities that we need to fix by means of some conventional procedure.

\subsection{Linking number}

Let us consider first the case $M_{3} \sim S^{3}$. Let $C_{1}$ and $C_{2}$ be two non-intersecting oriented knots in $S^{3}$ and let $\eta_{1}$ and $\eta_{2}$ the corresponding distributional 1-forms described in Section 2.4, one has

$$
\int_{S^{3}} \eta_{1} \wedge d \eta_{2}=\int_{S^{3}} \eta_{2} \wedge d \eta_{1}=\ell k\left(C_{1}, C_{2}\right)
$$

where $\ell k\left(C_{1}, C_{2}\right)$ denotes the linking number of $C_{1}$ and $C_{2}$, which is an integer valued ambient isotopy invariant. In fact, $\eta_{1} \wedge d \eta_{2}$ represents an intersection form counting how many times $C_{2}$ intersects the Seifert surface associated with $C_{1}$ (see also, for instance, $[28,29]$ ). Let $\left[\eta_{1}\right]$ and $\left[\eta_{2}\right]$ denote the $\mathrm{DB}$ classes which are associated with $\eta_{1}$ and $\eta_{2}$; since the linking number is an integer, one finds

$$
\left.\exp \left\{2 i \pi \int_{S^{3}}\left[\eta_{1}\right] *\left[\eta_{2}\right]\right\}=\exp \left\{2 i \pi \int_{S^{3}}\left[\eta_{2}\right] *\left[\eta_{1}\right]\right\}=\exp \left\{2 i \pi \int_{S^{3}} \eta_{1} \wedge d \eta_{2}\right]\right\}=1 .
$$

Equations (3.1) and (3.2) show that the product $\left[\eta_{1}\right] *\left[\eta_{2}\right]$ is well defined and just corresponds to the trivial class

$$
\left[\eta_{1}\right] *\left[\eta_{2}\right]=[0] \in \widetilde{H}_{D}^{3}\left(S^{3}, \mathbb{Z}\right)
$$

In the next sections, we shall encounter the linking number in the DB cohomology context in the following form. Let $x$ be a real number, since $\eta_{2}$ is globally defined in $S^{3}$, the 1 -form $x \eta_{2}$ is also globally defined. Let us denote by $\left[x \eta_{2}\right]$ the DB class which is represented by the form $x \eta_{2}$. One has

$$
\exp \left\{2 i \pi \int_{S^{3}}\left[\eta_{1}\right] *\left[x \eta_{2}\right]\right\}=\exp \left\{2 i \pi \int_{S^{3}} \eta_{1} \wedge d\left(x \eta_{2}\right)\right\}=\exp \left\{2 i \pi x \ell k\left(C_{1}, C_{2}\right)\right\} .
$$

\subsection{Framing}

Let $\eta_{C}$ be the distributional 1-form which is associated with the oriented knot $C \subset S^{3}$; for a single knot, the expression of the self-linking number

$$
\int_{S^{3}} \eta_{C} \wedge d \eta_{C}
$$


is not well defined because the self-intersection form $\eta_{C} \wedge d \eta_{C}$ has ambiguities. This means that, similarly to what happens with the product of distributions, at the level of the class $\left[\eta_{C}\right] \in \widetilde{H}_{D}^{1}\left(S^{3}, \mathbb{Z}\right)$, the product $\left[\eta_{C}\right] *\left[\eta_{C}\right]$ is not well defined a priori.

As shown in equations (2.14) and (2.15), $\left[\eta_{C}\right]$ can be determined by means of the $\varepsilon \rightarrow 0$ limit of $\left[\eta_{\varepsilon}\right] \in H_{D}^{1}\left(M_{3}, \mathbb{Z}\right)$. One could then try to define the product $\left[\eta_{C}\right] *\left[\eta_{C}\right]$ by means of the same limit

$$
\lim _{\varepsilon \rightarrow 0} \int_{S^{3}}\left[\eta_{\varepsilon}\right] *\left[\eta_{\varepsilon}\right]=\int_{S^{3}}\left[\eta_{C}\right] *\left[\eta_{C}\right]
$$

Unfortunately, the limit (3.6) does not exist, because the value that one obtains for the integral (3.6) in the $\varepsilon \rightarrow 0$ limit nontrivially depends on the way in which $\left[\eta_{\varepsilon}\right]$ approaches $\left[\eta_{C}\right]$. This problem will be solved by the introduction of the framing procedure, which ultimately specifies how $\left[\eta_{\varepsilon}\right]$ approaches $\left[\eta_{C}\right]$. One should note that the ambiguities entering the integral (3.5) and the limit (3.6) also appear in the Gauss integral

$$
\frac{1}{4 \pi} \oint_{C} d x^{\mu} \oint_{C} d y^{\nu} \epsilon_{\mu \nu \rho} \frac{(x-y)^{\rho}}{|x-y|^{3}}
$$

which corresponds to the self-linking number. A direct computation [30] shows that the value of the integral (3.7) is a real number which is not invariant under ambient isotopy transformations; in fact, it can be smoothly modified by means of smooth deformations of the $\operatorname{knot} C$ in $S^{3}$. In order to remove all ambiguities and define the product $\left[\eta_{C}\right] *\left[\eta_{C}\right]$, we shall adopt the framing procedure [29, 31], which is also used for giving a topological meaning to the self-linking number.

Definition 3.1. A solid torus is a space homeomorphic to $S^{1} \times D^{2}$, where $D^{2}$ is a two dimensional disc; in the complex plane, $D^{2}$ can be represented by the set $\{z$, with $|z| \leq 1\}$. Consider now an oriented knot $C \subset S^{3}$; a tubular neighbourhood $V_{C}$ of $C$ is a solid torus embedded in $S^{3}$ whose core is $C$. A given homeomorphism $h: S^{1} \times D^{2} \rightarrow V_{C}$ is called a framing for $C$. The image of the standard longitude $h\left(S^{1} \times 1\right)$ represents a knot $C_{f} \subset S^{3}$, also called the framing of $C$, which lies in a neighbourhood of $C$ and whose orientation is fixed to agree with the orientation of $C$. Up to isotopy transformations, the homeonorphism $h$ is specified by $C_{f}$.

Clearly, the thickness of the tubular neighbourhood $V_{C}$ of $C$ is chosen to be sufficiently small so that, in the presence of several link components for instance, any knot different from $C$ belongs to the complement of $V_{C} \subset S^{3}$.

For each framed knot $C$, with framing $C_{f}$, the self-linking number of $C$ is defined to be $\ell k\left(C, C_{f}\right)$

$$
\int_{S^{3}} \eta_{C} \wedge d \eta_{C} \equiv \int_{S^{3}} \eta_{C} \wedge d \eta_{C_{f}}=\ell k\left(C, C_{f}\right)
$$

Definition 3.2. In agreement with equation (3.8), one can consistently define the product $\left[\eta_{C}\right] *\left[\eta_{C}\right]$ as

$$
\left[\eta_{C}\right] *\left[\eta_{C}\right] \equiv\left[\eta_{C}\right] *\left[\eta_{C_{f}}\right]
$$

Definition (3.9) together with equations (3.8) and (3.3) imply that, for each framed knot $C$ (in $S^{3}$ ), the product $\left[\eta_{C}\right] *\left[\eta_{C}\right]$ is well defined and corresponds to the trivial class

$$
\left[\eta_{C}\right] *\left[\eta_{C}\right]=[0] \in \widetilde{H}_{D}^{3}\left(S^{3}, \mathbb{Z}\right) .
$$


Remark 3.1. The product $\left[\eta_{C}\right] *\left[\eta_{C}\right]$ also admits a definition which differs from equation (3.9) but, as far as the computation of the Chern-Simons observables is concerned, is equivalent to equation (3.9). Instead of dealing with a tubular neighbourhood $V_{C}$ with sufficiently small but finite thickness, one can define a limit in which the transverse size of the neighbourhood $V_{C}$ vanishes. Let $\rho>0$ be the size of the diameter of the tubular neighbourhood $V_{C}(\rho)$ of the knot $C ; \rho$ is defined with respect to some (topology compatible) metric $g$. The homeomorphism $h(\rho): S^{1} \times D^{2} \rightarrow V_{C}(\rho)$ is assumed to depend smoothly on $\rho$. Then, the corresponding framing knot $C_{f}(\rho)$ also smoothly depends on $\rho$. Consequently, the linking number $\ell k\left(C, C_{f}(\rho)\right)$ does not depend on the value of $\rho$ and it will be denoted by $\ell k\left(C, C_{f}\right)$. It should be noted that $\ell k\left(C, C_{f}\right)$ does not depend on the choice of the metric $g$. In the $\rho \rightarrow 0$ limit, the solid torus $V_{C}(\rho)$ shrinks to its core $C$ and the framing $C_{f}(\rho)$ goes to $C$. One can then define $\eta_{C} \wedge d \eta_{C}$ according to

$$
\int_{S^{3}} \eta_{C} \wedge d \eta_{C} \equiv \lim _{\rho \rightarrow 0} \int_{S^{3}} \eta_{C} \wedge d \eta_{C_{f}(\rho)}=\lim _{\rho \rightarrow 0} \ell k\left(C, C_{f}(\rho)\right)=\ell k\left(C, C_{f}\right) .
$$

In agreement with equation (3.10), one can put

$$
\left[\eta_{C}\right] *\left[\eta_{C}\right] \equiv \lim _{\rho \rightarrow 0}\left[\eta_{C}\right] *\left[\eta_{C_{f}(\rho)}\right] .
$$

Remark 3.2. The definition (3.9) of the DB product $\left[\eta_{C}\right] *\left[\eta_{C}\right]$ is consistent with equations (3.2)-(3.4) and is topologically well defined. In fact, in the case of an oriented framed link $L$ with $N$ components $\left\{C_{1}, C_{2}, \ldots, C_{N}\right\}$ the corresponding canonical class $\left[\eta_{L}\right] \in \widetilde{H}_{D}^{1}\left(S^{3}, \mathbb{Z}\right)$ is equivalent to the sum of the classes which are associated with the single components, i.e. $\left[\eta_{L}\right]=$ $\sum_{j}\left[\eta_{j}\right]$. Thus one finds

$$
\left[\eta_{L}\right] *\left[\eta_{L}\right]=\sum_{j}\left[\eta_{j}\right] *\left[\eta_{j}\right]+2 \sum_{i<j}\left[\eta_{i}\right] *\left[\eta_{j}\right]
$$

The framing procedure which is used to define the DB product $\left[\eta_{L}\right] *\left[\eta_{L}\right]$ guarantees that, if one integrates the 3-forms entering expression (3.12), the result does not depend on the particular choice of the Seifert surface which is used to (locally) define the distributional forms associated with $L$. This means that the framing procedure preserves both gauge invariance and ambient isotopy invariance.

Remark 3.3. In order to define the extension of the DB product to distributional DB classes, one could try to start from equation (2.11). In this case, the product of the DB representatives of two cycles (2.11) would only contain local integral chains and the cup product $\cup$ would just reduce to the intersection number of such integral chains (once these chains have been placed into transverse position, which is always possible because of the freedom in the choice of the DB cocycles representing a given DB class). Accordingly, the extension of the product to the distributional case would only produce integral chains and eventually integers in the integrals. Finally, by using smooth approximations of the cycles within (2.11) and then performing the limits, as described above in equation (3.11), one would obtain the same result. Note that, in this last approach, the limit would be performed with the linking number $\ell k\left(C, C_{f}\right)$ fixed. This is similar to the definition of the charge density of a charged point particle by taking the limit $r \rightarrow 0$ of a uniformly charged sphere of radius $r$ while keeping the total charge of the sphere fixed, which leads to the well-known Dirac delta-distribution.

Knots or links can be framed in any oriented 3-manifold $M_{3}$. In order to preserve the topological properties of the pairing $\widetilde{H}_{D}^{1}\left(S^{3}, \mathbb{Z}\right) \times \widetilde{H}_{D}^{1}\left(S^{3}, \mathbb{Z}\right) \rightarrow \widetilde{H}_{D}^{3}\left(S^{3}, \mathbb{Z}\right)$ which is defined by means of framing in $S^{3}$, we shall extend the framing procedure to the case of a generic 3-manifold $M_{3}$ by extending the validity of properties (3.3) and (3.9). 
Definition 3.3. If $\left[\eta_{1}\right]$ and $\left[\eta_{2}\right]$ are the classes in $\widetilde{H}_{D}^{1}\left(M_{3}, \mathbb{Z}\right)$ which are canonically associated with the oriented nonintersecting knots $C_{1}$ and $C_{2}$ in $M_{3}$, in agreement with equation (3.3) we shall eliminate the (possible) ambiguities of the product $\left[\eta_{1}\right] *\left[\eta_{2}\right]$ in such a way that

$$
\left[\eta_{1}\right] *\left[\eta_{2}\right]=[0] \in \widetilde{H}_{D}^{3}\left(M_{3}, \mathbb{Z}\right) .
$$

Consequently, for each oriented framed knot $C \subset M_{3}$ with framing $C_{f}$, we shall use the definition

$$
\left[\eta_{C}\right] *\left[\eta_{C}\right] \equiv\left[\eta_{C}\right] *\left[\eta_{C_{f}}\right]=[0] \in \widetilde{H}_{D}^{3}\left(M_{3}, \mathbb{Z}\right) .
$$

Remark 3.4. Definition (3.14) can also be understood by starting from equation (2.11) and by using the same arguments that have been presented in the case $M_{3} \sim S^{3}$. Let us point out that, unlike the $S^{3}$ case, for generic $M_{3}$ one finds directly equation (3.14) without the validity of some intermediate relations like equation (3.8), which may not be well defined for $M_{3} \nsim S^{3}$.

\section{Abelian Chern-Simons field theory}

\subsection{Action functional}

If one uses the Cech-de Rham double complex to describe DB classes, it can easily be shown that the first component of a DB product of a $U(1)$-connection $A$ with itself is given by $A \wedge d A$ or, more precisely, it is given by the collection of all these products taken in the open sets of a good cover of $M_{3}$. This means that the expression of the Chern-Simons lagrangian of a $U$ (1)connection $A$ can be understood as a DB class which coincides with the "DB square" of the class of $A$. Let $[A]$ denote the DB class associated to the $U(1)$-connection $A$, the Chern-Simons functional $S_{C S}$ is given by

$$
S_{C S}=\int_{M_{3}}[A] *[A]
$$

By definition of the DB cohomology, the Chern-Simons action $S_{C S}$ is an element of $\mathbb{R} / \mathbb{Z}$ and then it is defined modulo integers. Consequently, in the functional measure of the path-integral, the phase factor which is induced by the action has to be of the type

$$
\exp \left\{2 i \pi k S_{C S}\right\}=\exp \left\{2 i \pi k \int_{M_{3}}[A] *[A]\right\}
$$

where the coupling constant $k$ must be a nonvanishing integer

$$
k \in \mathbb{Z}, \quad k \neq 0 .
$$

A modification of the orientation of $M_{3}$ is equivalent to the replacement $k \rightarrow-k$.

\subsection{Observables}

The observables that we shall consider are given by the expectation values of the Wilson line operators $W(L)$ associated with links $L$ in $M_{3}$. An oriented coloured and framed link $L \subset M_{3}$ with $N$ components is the union of non-intersecting knots $\left\{C_{1}, C_{2}, \ldots, C_{N}\right\}$ in $M_{3}$, where each knot $C_{j}$ (with $j=1,2, \ldots, N$ ) is oriented and framed, and its colour is represented by an integer charge $q_{j} \in \mathbb{Z}$. For any given DB class $[A]$, the classical expression of $W(L)$ is given by

$$
W(L)=\prod_{j=1}^{N} \exp \left\{2 i \pi q_{j} \int_{C_{j}}[A]\right\}=\exp \left\{2 i \pi \sum_{j} q_{j} \int_{C_{j}}[A]\right\},
$$


which actually corresponds to the pairing (2.6)

$$
W(L)=\exp \left\{2 i \pi \int_{L}[A]\right\} \equiv \exp \left\{2 i \pi\langle[A], L\rangle_{1}\right\} .
$$

Once more, each factor

$$
\exp \left\{2 i \pi q_{j} \int_{C_{j}}[A]\right\},
$$

which appears in expression (4.1), is well defined if and only if $q_{j} \in \mathbb{Z}$; that is why the charges associated with knots must take integer values. A modification of the orientation of the knot $C_{j}$ is equivalent to the replacement $q_{j} \rightarrow-q_{j}$. Obviously, any link component with colour $q=0$ can be eliminated.

Remark 4.1. The classical expression (4.1) does not depend on the framing of the knots $\left\{C_{j}\right\}$; however, only for framed links are the Wilson line operators well defined. The point is that, in the quantum Chern-Simons field theory, the field components correspond to distributional valued operators, and the Wilson line operators are formally defined by expression (4.1) together with a set of specified rules which must be used to remove possible ambiguities in the computation of the expectation values. In the operator formalism, these ambiguities are related to the product of field operators in the same point [32, 33] and they are eliminated by means of a framing procedure. In the path-integral approach, we shall see that all the ambiguities are related to the definition of the pairing $\widetilde{H}_{D}^{1}\left(M_{3}, \mathbb{Z}\right) \times \widetilde{H}_{D}^{1}\left(M_{3}, \mathbb{Z}\right) \rightarrow \widetilde{H}_{D}^{3}\left(M_{3}, \mathbb{Z}\right)$; as it has been discussed in Section 3, we shall use the framing of the link components to eliminate all ambiguities by means of the definition (3.14).

Remark 4.2. In equations (4.1) and (4.2), we have used the same symbol to denote knots and their homological representatives because a canonical correspondence [28] between them always exists. At the classical level, for any integer $q$ one can identify the 1-cycle $q C \in Z_{1}(M)$ with the $q$-fold covering of the cycle $C$ or the $q$-times product of $C$ with itself. At the quantum level, this equivalence may not be valid when it is applied to the Wilson line operators because of ambiguities in the definition of composite operators; so, in order to avoid inaccuracies, we will always refer to Wilson line operators defined for oriented coloured and framed knots or links.

Definition 4.1. For each link component $C_{j}$, let $\left[\eta_{j}\right] \in \widetilde{H}_{D}^{1}\left(M_{3}, \mathbb{Z}\right)$ be the DB class such that

$$
\exp \left\{2 i \pi q_{j} \int_{C_{j}}[A]\right\}=\exp \left\{2 i \pi q_{j} \int_{M_{3}}[A] *\left[\eta_{j}\right]\right\} .
$$

With the definition

$$
\left[\eta_{L}\right]=\sum_{j} q_{j}\left[\eta_{j}\right]
$$

one has

$$
\exp \left\{2 i \pi \int_{M_{3}}[A] *\left[\eta_{L}\right]\right\}=\exp \left\{2 i \pi \sum_{j} q_{j} \int_{M_{3}}[A] *\left[\eta_{j}\right]\right\} .
$$

The expectation values of the Wilson line operators can be written in the form

$$
\langle W(L)\rangle_{k} \equiv \frac{\int D[A] \exp \left\{2 i \pi k \int_{M_{3}}[A] *[A]\right\} W(L)}{\int D[A] \exp \left\{2 i \pi k \int_{M_{3}}[A] *[A]\right\}}
$$




$$
=\frac{\int D[A] \exp \left\{2 i \pi k \int_{M_{3}}[A] *[A]\right\} \exp \left\{2 i \pi \int_{M_{3}}[A] *\left[\eta_{L}\right]\right\}}{\int D[A] \exp \left\{2 i \pi k \int_{M_{3}}[A] *[A]\right\}}
$$

and our main purpose is to show how to compute them for arbitrary link $L$.

Remark 4.3. In the DB cohomology approach, the functional integration (4.4) locally corresponds to a sum over 1-form modulo forms with integer periods. So, the space of classical field configurations which are factorized out by gauge invariance is in general larger than the standard group of local gauge transformations. It should be noted that this enlarged gauge symmetry perfectly fits the assumption that the expectation values (4.4) form a complete set of observables. In the DB cohomology interpretation of the functional integral for the quantum Chern-Simons field theory, this enlargement of the "symmetry group" represents one of the main conceptual improvements with respect to the standard formulation of gauge theories and, as we shall show, allows for a description of the functional space structure in terms of the homology groups of the manifold $M_{3}$.

\subsection{Properties of the functional measure}

The sum over the DB classes $\int D[A]$ corresponds to a gauge-fixed functional integral in ordinary quantum field theory, where one has to sum over the gauge orbits in the space of connections. In the path-integral, smooth fields configurations or finite-action configurations have zero measure [34, 35]; so, the functional integral (4.4) has to be understood as the functional integral in the appropriate extension or closure $\mathcal{H}_{D}^{1}\left(M_{3}, \mathbb{Z}\right)$ of the space $H_{D}^{1}\left(M_{3}, \mathbb{Z}\right)$, with $\widetilde{H}_{D}^{1}\left(M_{3}, \mathbb{Z}\right) \subset$ $\mathcal{H}_{D}^{1}\left(M_{3}, \mathbb{Z}\right)$ and, more generaly, with $\operatorname{Hom}\left(H_{D}^{1}(M, \mathbb{Z}), S^{1}\right) \subset \mathcal{H}_{D}^{1}\left(M_{3}, \mathbb{Z}\right)$. In order to guarantee the consistency of the functional integral and its correspondence with ordinary gauge theories, we assume that the quantum measure has the following two properties.

(M1) The space $\mathcal{H}_{D}^{1}\left(M_{3}, \mathbb{Z}\right)$ inherits its structure from $H_{D}^{1}\left(M_{3}, \mathbb{Z}\right)$ in agreement with sequence (2.5).

Equation (2.5) then implies that the sum over DB classes is locally equivalent to a sum over $\Omega^{1}\left(M_{3}\right) / \Omega_{\mathbb{Z}}^{1}\left(M_{3}\right)$, i.e. a sum over 1-forms modulo generalized gauge transformations.

(M2) The functional measure is translational invariant.

This implies in particular that, for any $[\omega] \in \widetilde{H}_{D}^{1}\left(M_{3}, \mathbb{Z}\right)$, the quadratic measure

$$
d \mu_{k}([A]) \equiv D[A] \exp \left\{2 i \pi k \int_{M_{3}}[A] *[A]\right\}
$$

satisfies the equation

$$
d \mu_{k}([A]+[\omega])=d \mu_{k}([A]) \exp \left\{4 i \pi k \int_{M_{3}}[A] *[\omega]+2 i \pi k \int_{M_{3}}[\omega] *[\omega]\right\},
$$

which looks like a Cameron-Martin formula (see for instance [36] and references therein).

Equation (4.6) will be used extensively in our computations. The importance of generalized Wiener measures in the functional integral - which necessarily imply the validity of the CameronMartin property - and of the singular connections was also stressed in the articles [37] and [38] in which, however, the space of the functional integral is supposed to coincide with the space of the classes of smooth connections on a fixed $U(1)$-bundle over $M_{3}$.

In the computation of the observables (4.4), we shall not use perturbation theory; only properties (M1) and (M2) of the functional measure will be utilized. We shall now derive the main properties of the observables of the Abelian Chern-Simons theory which are valid for any 3-manifold $M_{3}$. 


\subsection{Colour periodicity}

The colour of each oriented knot or link component $C \subset M_{3}$ is specified by the value of its associated charge $q \in \mathbb{Z}$. For fixed nonvanishing value of the coupling constant $k$, the expectation values (4.4) are invariant under the substitution $q \rightarrow q+2 k$, where $q$ is the charge of a generic link component. Consequently, one has

Proposition 4.1. For fixed integer $k$, the colour space is given by $\mathbb{Z}_{2 k}$ which coincides with the space of residue classes of integers $\bmod 2 k$.

Proof. Let $\left\{q_{j}\right\}$ be the charges which are associated with the components $\left\{C_{j}\right\}(j=1,2, \ldots, N)$ of the link $L$. With the notation (4.5), the expectation value $\langle W(L)\rangle_{k}$ shown in equation (4.4) can be written as

$$
\langle W(L)\rangle_{k}=\frac{\int d \mu_{k}([A]) \exp \left\{2 i \pi \sum_{j} q_{j} \int_{M_{3}}[A] *\left[\eta_{j}\right]\right\}}{\int d \mu_{k}([A])} .
$$

Property (M2) implies that, with the substitution $[A] \rightarrow[A]+\left[\eta_{1}\right]$, the numerator of expression (4.7) becomes

$$
\begin{aligned}
& \int d \mu_{k}([A]) \exp \left\{2 i \pi \sum_{j} q_{j} \int_{M_{3}}[A] *\left[\eta_{j}\right]\right\}=\int d \mu_{k}([A]) \exp \left\{2 i \pi \sum_{j} q_{j}^{\prime} \int_{M_{3}}[A] *\left[\eta_{j}\right]\right\} \\
& \quad \times \exp \left\{2 i \pi k \int_{M_{3}}\left[\eta_{1}\right] *\left[\eta_{1}\right]\right\} \exp \left\{2 i \pi \sum_{j} q_{j} \int_{M_{3}}\left[\eta_{1}\right] *\left[\eta_{j}\right]\right\},
\end{aligned}
$$

where $q_{j}^{\prime}=q_{j}+2 k \delta_{j 1}$. In agreement with equation (3.13), for $j \neq 1$ one has $\left[\eta_{1}\right] *\left[\eta_{j}\right] \simeq[0] \in$ $\widetilde{H}_{D}^{3}\left(M_{3}, \mathbb{Z}\right)$, and then

$$
\exp \left\{2 i \pi q_{j} \int_{M_{3}}\left[\eta_{1}\right] *\left[\eta_{j}\right]\right\}=1
$$

Similarly, in agreement with equation (3.14), by means of the framing procedure one obtains $\left[\eta_{1}\right] *\left[\eta_{1}\right] \simeq[0] \in \widetilde{H}_{D}^{3}\left(M_{3}, \mathbb{Z}\right)$, and then

$$
\exp \left\{2 i \pi\left(q_{1}+k\right) \int_{M_{3}}\left[\eta_{1}\right] *\left[\eta_{1}\right]\right\}=1 \text {. }
$$

Consequently, the numerator of expression (4.7) can be written as

$$
\begin{aligned}
& \int d \mu_{k}([A]) \exp \left\{2 i \pi \sum_{j} q_{j} \int_{M_{3}}[A] *\left[\eta_{j}\right]\right\} \\
& =\int d \mu_{k}([A]) \exp \left\{2 i \pi \sum_{j} q_{j}^{\prime} \int_{M_{3}}[A] *\left[\eta_{j}\right]\right\},
\end{aligned}
$$

which proves that, for fixed $k$, the expectation values (4.4) are invariant under the substitution $q_{1} \rightarrow q_{1}+2 k$, where $q_{1}$ is the charge of the link component $C_{1}$. 


\subsection{Ambient isotopy invariance}

Two oriented framed coloured links $L$ and $L^{\prime}$ in $M_{3}$ are ambient isotopic if $L$ can be smoothly connected with $L^{\prime}$ in $M_{3}$.

Proposition 4.2. The Chern-Simons expectation values (4.4) are invariants of ambient isotopy for framed links.

Proof. Suppose that two oriented coloured framed links $L$ and $L^{\prime}$ are ambient isotopic in $M_{3}$. The link $L$ has components $\left\{C_{1}, C_{2}, \ldots, C_{N}\right\}$ with colours $\left\{q_{1}, q_{2}, \ldots, q_{N}\right\}$; whereas the link $L^{\prime}$ has components $\left\{C_{1}^{\prime}, C_{2}, \ldots, C_{N}\right\}$ with colours $\left\{q_{1}, q_{2}, \ldots, q_{N}\right\}$, so that

$$
\left[\eta_{L}\right]=q_{1}\left[\eta_{1}\right]+\sum_{j \neq 1} q_{j}\left[\eta_{j}\right], \quad\left[\eta_{L^{\prime}}\right]=q_{1}\left[\eta_{1}^{\prime}\right]+\sum_{j \neq 1} q_{j}\left[\eta_{j}\right]
$$

where the class $\left[\eta_{1}\right]$ refers to the knot $C_{1} \subset M_{3}$ and $\left[\eta_{1}^{\prime}\right]$ is associated to the knot $C_{1}^{\prime} \subset M_{3}$.

Let $\tau:[0,1] \rightarrow C_{1}(\tau) \subset M_{3}$ be the isotopy which connects $C_{1}$ with $C_{1}^{\prime}$ in $M_{3}$, with $C_{1}(0)=C_{1}$ and $C_{1}(1)=C_{1}^{\prime}$. We shall denote by $\Sigma \subset M_{3}$ the 2 -dimensional surface which has support on $\left\{C_{1}(\tau) \subset M_{3} ; 0 \leq \tau \leq 1\right\}$; because of the freedom in the choice of $\tau$ within the same ambient isotopy class, it is assumed that $\Sigma$ is well defined and presents no singularities. $\Sigma$ belongs to the complement of the link components $\left\{C_{2}, C_{3}, \ldots, C_{N}\right\}$ in $M_{3}$ and one can introduce an orientation on $\Sigma$ in such a way that its oriented boundary is given by $\partial \Sigma=C_{1}^{\prime} \cup C_{1}^{-1}$, where $C_{1}^{-1}$ denotes the knot $C_{1}$ with reversed orientation.

The distributional 1-form $\eta_{\Sigma}$, which is associated with $\Sigma$, is globally defined in $M_{3}$ and satisfies

$$
d \eta_{\Sigma}=d \eta_{1}^{\prime}-d \eta_{1}
$$

where, with a small abuse of notation, $d \eta_{1}$ and $d \eta_{1}^{\prime}$ denote the integration currents of $C_{1}$ and $C_{1}^{\prime}$ respectively. For $j \neq 1$ one finds

$$
\int_{M_{3}} \eta_{\Sigma} \wedge d \eta_{j}=0
$$

because the link components $\left\{C_{2}, C_{3}, \ldots, C_{N}\right\}$ do not intersect the surface $\Sigma$. Moreover, according to the framing procedure, the orientation of $\Sigma$ implies

$$
\int_{M_{3}} \eta_{\Sigma} \wedge\left(d \eta_{1}^{\prime}+d \eta_{1}\right)=\int_{C_{1 f}^{\prime}} \eta_{\Sigma}+\int_{C_{1 f}} \eta_{\Sigma}=0,
$$

where $C_{1 f}^{\prime}$ denotes the framing of $C_{1}^{\prime}$ and $C_{1 f}$ represents the framing of $C_{1}$. Since $\eta_{\Sigma}$ is globally defined in $M_{3}$, the 1 -form $x \eta_{\Sigma}$ (with $x=\left(q_{1} / 2 k\right) \in \mathbb{R}$ ) is also globally defined. Let $\left[x \eta_{\Sigma}\right] \in$ $\widetilde{H}_{D}^{1}\left(M_{3}, \mathbb{Z}\right)$ be the DB class which can be represented by the 1 -form $x \eta_{\Sigma}$; by construction, one has

$$
\begin{aligned}
& \exp \left\{4 i \pi k \int_{M_{3}}[A] *\left[\left(q_{1} / 2 k\right) \eta_{\Sigma}\right]\right\} \\
& =\exp \left\{2 i \pi q_{1} \int_{M_{3}}[A] *\left[\eta_{1}^{\prime}\right]\right\} \exp \left\{-2 i \pi q_{1} \int_{M_{3}}[A] *\left[\eta_{1}\right]\right\} .
\end{aligned}
$$

The expectation value $\langle W(L)\rangle_{k}$ is given by

$$
\langle W(L)\rangle_{k}=\frac{\int d \mu_{k}([A]) \exp \left\{2 i \pi \int_{M_{3}}[A] *\left[\eta_{L}\right]\right\}}{\int d \mu_{k}([A])} .
$$


Equation (4.12) and property (M2) imply that, with the substitution $[A] \rightarrow[A]+\left[x \eta_{\Sigma}\right]$, the numerator of expression (4.13) can be written as

$$
\begin{aligned}
& \int d \mu_{k}([A]) \exp \left\{2 i \pi \int_{M_{3}}[A] *\left[\eta_{L^{\prime}}\right]\right\} \\
& \quad \times \exp \left\{2 i \pi k \int_{M_{3}}\left[x \eta_{\Sigma}\right] *\left[x \eta_{\Sigma}\right]\right\} \exp \left\{2 i \pi \int_{M_{3}}\left[x \eta_{\Sigma}\right] *\left[\eta_{L}\right]\right\} .
\end{aligned}
$$

By using the relations

$$
\begin{aligned}
& \exp \left\{2 i \pi k \int_{M_{3}}\left[x \eta_{\Sigma}\right] *\left[x \eta_{\Sigma}\right]\right\}=\exp \left\{\left(i \pi q_{1}^{2} / 2 k\right) \int_{M_{3}} \eta_{\Sigma} \wedge\left(d \eta_{1}^{\prime}-d \eta_{1}\right)\right\}, \\
& \exp \left\{2 i \pi \int_{M_{3}}\left[x \eta_{\Sigma}\right] *\left[\eta_{L}\right]\right\}=\exp \left\{\left(i \pi q_{1}^{2} / k\right) \int_{M_{3}} \eta_{\Sigma} \wedge d \eta_{1}\right\} \\
& \quad \times \exp \left\{\left(i \pi q_{1} / k\right) \sum_{j \neq 1} q_{j} \int_{M_{3}} \eta_{\Sigma} \wedge d \eta_{j}\right\},
\end{aligned}
$$

and equations (4.9)-(4.11), one finds that the numerator of expression (4.13) assumes the form

$$
\int d \mu_{k}([A]) \exp \left\{2 i \pi \int_{M_{3}}[A] *\left[\eta_{L^{\prime}}\right]\right\} \text {. }
$$

Consequently, the expectation values of the Wilson line operators associated with the links $L$ and $L^{\prime}$, entering equation (4.8), are equal. The same argument, applied to all the link components, implies that, for any two ambient isotopic links $L$ and $L^{\prime}$, one has

$$
\langle W(L)\rangle_{k}=\left\langle W\left(L^{\prime}\right)\right\rangle_{k}
$$

This concludes the proof.

\subsection{Satellite relations}

For the oriented framed knot $C \subset M_{3}$, let the homeomorphism $h: S^{1} \times D^{2} \rightarrow V_{C}$ be the framing of $C$, where $V_{C}$ is a a tubular neighbourhood of $C$. Let us represent the disc $D^{2}$ by the set $\{z$, with $|z| \leq 1\}$ of the complex plane. The framing $C_{f}$ of $C$ is given by $h\left(S^{1} \times 1\right)$, whereas one can always imagine that the knot $C$ just corresponds to $h\left(S^{1} \times 0\right)$. Let $P$ be a link in the solid torus $S^{1} \times D^{2}$; if one replaces the knot $C \subset M_{3}$ by $h(P) \subset M_{3}$ one obtains the satellite of $C$ which is defined by the pattern link $P$.

Definition 4.2. Let $B \subset S^{1} \times D^{2}$ be the oriented link with two components $\left\{B_{1}, B_{2}\right\}$ given by $B_{1}=\left(S^{1} \times 0\right) \subset S^{1} \times D^{2}$ and $B_{2}=\left(S^{1} \times 1 / 2\right) \subset S^{1} \times D^{2}$. For any oriented framed knot $C \subset M_{3}$, let us denote by $C^{(2)} \in M_{3}$ the satellite of $C$ with is obtained by means of the pattern link $B$. The two oriented components $\left\{K_{1}, K_{2}\right\}$ of $C^{(2)}$ are given by $K_{1}=h\left(B_{1}\right)$ and $K_{2}=h\left(B_{2}\right)$. Let us introduce a framing for the components of the link $C^{(2)}$; the knot $K_{1}$ has framing $K_{1 f}=h\left(S^{1} \times 1 / 4\right)$ and the knot $K_{2}$ has framing $K_{2 f}=h\left(S^{1} \times 3 / 4\right)$.

By construction, the satellite $C^{(2)}$ of $C$ is an oriented framed link.

Proposition 4.3. Let $L$ and $\widetilde{L}$ be two oriented coloured framed links in $M_{3}$ in which $\widetilde{L}$ is obtained from $L=\left\{C_{1}, \ldots, C_{N}\right\}$ by substituting the component $C_{1}$, which has colour $q_{1} \in \mathbb{Z}$, with its satellite $C_{1}^{(2)}$ whose components $K_{1}$ and $K_{2}$ have colours $\widetilde{q}_{1}=q_{1} \pm 1$ and $\widetilde{q}_{2}=\mp 1$ respectively. Then, the corresponding Chern-Simons expectation values satisfy

$$
\langle W(L)\rangle_{k}=\langle W(\widetilde{L})\rangle_{k}
$$


Proof. Because of the ambient isotopy invariance of $\langle W(\widetilde{L})\rangle_{k}$, one can consider the limit in which the component $K_{1}$ approaches to $K_{2}$ and coincides with $K_{2}$. In this limit, for each field configuration (i.e. for each DB class) the associated holonomies $W\left(C_{1}\right)$ and $W\left(C_{1}^{(2)}\right)$ coincides. This means that, at the classical level, equality (4.14) is satisfied. Thus, we only need to consider possible ambiguities in the expectation value of the composite Wilson line operator $W\left(C_{1}^{(2)}\right)=W\left(K_{1}\right) W\left(K_{2}\right)$ in the $K_{1} \rightarrow K_{2}$ limit. In agreement with what we shall show in the following sections, we now assume that all the ambiguities which refer to composite Wilson line operators are eliminated by means of the framing procedure which is used to define the product $\left[\eta_{\widetilde{L}}\right] *\left[\eta_{\widetilde{L}}\right]$. According to the definition (4.3), one has

$$
\begin{aligned}
& {\left[\eta_{L}\right]=q_{1}\left[\eta_{1}\right]+\sum_{j=2}^{N} q_{j}\left[\eta_{j}\right]=q_{1}\left[\eta_{1}\right]+\left[\bar{\eta}_{L}\right],} \\
& {\left[\eta_{\widetilde{L}}\right]=\widetilde{q}_{1}\left[\eta_{K_{1}}\right]+\widetilde{q}_{2}\left[\eta_{K_{2}}\right]+\sum_{j=2}^{N} q_{j}\left[\eta_{j}\right]=\widetilde{q}_{1}\left[\eta_{K_{1}}\right]+\widetilde{q}_{2}\left[\eta_{K_{2}}\right]+\left[\bar{\eta}_{L}\right],}
\end{aligned}
$$

and then

$$
\begin{aligned}
{\left[\eta_{L}\right] *\left[\eta_{L}\right]=} & q_{1}^{2}\left[\eta_{C_{1}}\right] *\left[\eta_{C_{1}}\right]+2 q_{1}\left[\eta_{C_{1}}\right] *\left[\bar{\eta}_{L}\right]+\left[\bar{\eta}_{L}\right] *\left[\bar{\eta}_{L}\right] \\
{\left[\eta_{\widetilde{L}}\right] *\left[\eta_{\widetilde{L}}\right]=} & \left(\widetilde{q}_{1}\left[\eta_{K_{1}}\right]+\widetilde{q}_{2}\left[\eta_{K_{2}}\right]\right) *\left(\widetilde{q}_{1}\left[\eta_{K_{1}}\right]+\widetilde{q}_{2}\left[\eta_{K_{2}}\right]\right) \\
& +2\left(\widetilde{q}_{1}\left[\eta_{K_{1}}\right]+\widetilde{q}_{2}\left[\eta_{K_{2}}\right]\right) *\left[\bar{\eta}_{L}\right]+\left[\bar{\eta}_{L}\right] *\left[\bar{\eta}_{L}\right] .
\end{aligned}
$$

As far as the computation of the Chern-Simons observables is concerned, ambient isotopy invariance and equality $q_{1}=\widetilde{q}_{1}+\widetilde{q}_{2}$ imply

$$
2 q_{1}\left[\eta_{C_{1}}\right] *\left[\bar{\eta}_{L}\right]=2\left(\widetilde{q}_{1}\left[\eta_{K_{1}}\right]+\widetilde{q}_{2}\left[\eta_{K_{2}}\right]\right) *\left[\bar{\eta}_{L}\right],
$$

moreover, by construction of the satellite $C_{1}^{(2)}$ and the definition (3.14), one also finds

$$
q_{1}^{2}\left[\eta_{C_{1}}\right] *\left[\eta_{C_{1}}\right]=\left(\widetilde{q}_{1}\left[\eta_{K_{1}}\right]+\widetilde{q}_{2}\left[\eta_{K_{2}}\right]\right) *\left(\widetilde{q}_{1}\left[\eta_{K_{1}}\right]+\widetilde{q}_{2}\left[\eta_{K_{2}}\right]\right) .
$$

Therefore, as far as the computation of the Chern-Simons observables is concerned, one can replace $\left[\eta_{L}\right] *\left[\eta_{L}\right]$ by $\left[\eta_{\widetilde{L}}\right] *\left[\eta_{\widetilde{L}}\right]$, and then $\langle W(L)\rangle_{k}=\langle W(\widetilde{L})\rangle_{k}$.

Definition 4.3. In agreement with Proposition 4.3, for any oriented coloured framed link $L \subset$ $M_{3}$, one can replace recursively all the link components which have colour given by $q \neq \pm 1$ by their satellites constructed with the pattern link $B$, in such a way that the resulting $\operatorname{link} \bar{L} \subset M_{3}$ has the following property: each oriented framed component of $\bar{L}$ has colour which is specified by a charge $q=+1$ or $q=-1$. Remember that, for each link component $C$, the sign of the associated charge $q$ is defined with respect to the orientation of $C$. So, with a suitable choice of the orientation of the link components, all the link components of $\bar{L}$ have charges +1 . For each link $L \subset M_{3}$, the corresponding link $\bar{L} \subset M_{3}$ will be called the simplicial satellite of $L$ and, as a consequence of Proposition 4.3, one has

$$
\langle W(L)\rangle_{k}=\langle W(\bar{L})\rangle_{k}
$$

\section{$5 \quad$ Abelian Chern-Simons theory on $S^{3}$}

When $M_{3}=S^{3}$, the DB cohomology group satisfies $H_{D}^{1}\left(S^{3}, \mathbb{Z}\right) \simeq \Omega^{1}\left(S^{3}\right) / \Omega_{\mathbb{Z}}^{1}\left(S^{3}\right)$ and one has $\Omega^{1}\left(S^{3}\right) / \Omega_{\mathbb{Z}}^{1}\left(S^{3}\right)=\Omega^{1}\left(S^{3}\right) / d \Omega^{0}\left(S^{3}\right)$. Since in general the path-integral of the ChernSimons theory on $M_{3}$ locally corresponds to a sum over the space of 1-forms modulo forms 
with integer periods, it is convenient to introduce a new notation; with respect to the origin of $\Omega^{1}\left(S^{3}\right) / \Omega_{\mathbb{Z}}^{1}\left(S^{3}\right)$ that one can choose to correspond to the vanishing connection, an element of $\Omega^{1}\left(S^{3}\right) / \Omega_{\mathbb{Z}}^{1}\left(S^{3}\right)$ will be denoted by $[\alpha]$. So that, in agreement with property (M1), for any oriented coloured and framed link $L \subset S^{3}$ the expectation value (4.4) can be written as

$$
\begin{aligned}
\langle W(L)\rangle_{k} & =\frac{\int D[\alpha] \exp \left\{2 i \pi k \int_{S^{3}}[\alpha] *[\alpha]\right\} \exp \left\{2 i \pi \int_{S^{3}}[\alpha] *\left[\eta_{L}\right]\right\}}{\int D[\alpha] \exp \left\{2 i \pi k \int_{S^{3}}[\alpha] *[\alpha]\right\}} \\
& =\frac{\int d \mu_{k}([\alpha]) \exp \left\{2 i \pi \int_{S^{3}}[\alpha] *\left[\eta_{L}\right]\right\}}{\int d \mu_{k}([\alpha])}
\end{aligned}
$$

where $[\alpha] \in \Omega^{1}\left(S^{3}\right) / \Omega_{\mathbb{Z}}^{1}\left(S^{3}\right)$ and $\left[\eta_{L}\right] \in \widetilde{H}_{D}^{1}\left(M_{3}, \mathbb{Z}\right)$ denotes the class which is canonically associated with $L$. The integral (5.1) actually extends to $\mathcal{H}_{D}^{1}\left(S^{3}, \mathbb{Z}\right)$ which has to be understood as a suitable extension of $\Omega^{1}\left(S^{3}\right) / \Omega_{\mathbb{Z}}^{1}\left(S^{3}\right)$. We shall now compute the observable $\langle W(L)\rangle_{k}$ for arbitrary $\operatorname{link} L$.

Theorem 5.1. Let the oriented coloured and framed link components $\left\{C_{j}\right\}$ of the link $L$, with $j=1,2, \ldots, N$, have charges $\left\{q_{j}\right\}$ and framings $\left\{C_{j f}\right\}$. Then

$$
\langle W(L)\rangle_{k}=\exp \left\{-(2 i \pi / 4 k) \sum_{i j} q_{i} \mathbb{L}_{i j} q_{j}\right\},
$$

where the linking matrix $\mathbb{L}_{i j}$ is defined by

$$
\begin{aligned}
& \mathbb{L}_{i j}=\int_{S^{3}} \eta_{i} \wedge d \eta_{j}=\ell k\left(C_{i}, C_{j}\right), \quad \text { for } i \neq j, \\
& \mathbb{L}_{j j}=\int_{S^{3}} \eta_{j} \wedge d \eta_{j}=\ell k\left(C_{j}, C_{j f}\right) .
\end{aligned}
$$

Proof. Since $H^{2}\left(S^{3}, \mathbb{Z}\right)=0$, Poincaré duality implies that any 1-cycle on $S^{3}$ is homologically trivial. Equivalently, for each knot $C_{j}$ one can find an oriented Seifert surface $\Sigma_{j} \subset S^{3}$ such that $\partial \Sigma_{j}=C_{j}$ (in fact, there is an infinite number of topologically inequivalent Seifert surfaces) and one can then define a distributional 1-form $\eta_{j}$ (with support on $\Sigma_{j}$ ) which is globally defined in $S^{3}$. The distributional 1-form $\eta_{L}$ associated with the link $L$,

$$
\eta_{L}=\sum_{j} q_{j} \eta_{j}
$$

is globally defined in $S^{3}$ and, in the Chech-de Rham description of DB cocycles, the class $\left[\eta_{L}\right]$ can be represented by the sequence $\left(\eta_{L}, 0,0\right)$. The distributional 1-form

$$
\eta_{L} / 2 k=\sum_{j}\left(q_{j} / 2 k\right) \eta_{j}
$$

is also globally defined in $S^{3}$ and we shall denote by $\left[\eta_{L} / 2 k\right] \in \widetilde{H}_{D}^{1}\left(M_{3}, \mathbb{Z}\right)$ the DB class which, in the Chech-de Rham description of DB cocycles, is represented by the sequence $\left(\eta_{L} / 2 k, 0,0\right)$. It should be noted that the class $\left[\eta_{L} / 2 k\right]$ does not depend on the particular choice of the 1-form $\eta_{L}$ which represents $\left[\eta_{L}\right]$. (In turn, this implies that $\left[\eta_{L} / 2 k\right]$ does not depend on the particular choice of the Seifert surfaces.) In fact, any representative 1-form of $\left[\eta_{L}\right]$ can be written as $\eta_{L}+d \chi$ for some $\chi \in \Omega^{0}\left(S^{3}\right)$; therefore, for the corresponding class $\left[\left(\eta_{L}+d \chi\right) / 2 k\right]$ one finds

$$
\left[\left(\eta_{L}+d \chi\right) / 2 k\right]=\left[\eta_{L} / 2 k+d \chi / 2 k\right]=\left[\eta_{L} / 2 k\right]+[d(\chi / 2 k)]=\left[\eta_{L} / 2 k\right] .
$$


By construction, the class $\left[\eta_{L} / 2 k\right]$ satisfies the relation

$$
2 k\left[\eta_{L} / 2 k\right]=\left[\eta_{L}\right]
$$

therefore

$$
\exp \left\{4 i \pi k \int_{S^{3}}[\alpha] *\left[\eta_{L} / 2 k\right]\right\}=\exp \left\{2 i \pi \int_{S^{3}}[\alpha] *\left[\eta_{L}\right]\right\}
$$

In agreement with property (M2), by means of the substitution $[\alpha] \rightarrow[\alpha]-\left[\eta_{L} / 2 k\right]$ the numerator of expression (5.1) assumes the form

$$
\begin{gathered}
\int d \mu_{k}([\alpha]) \exp \left\{-4 i \pi k \int_{S^{3}}[\alpha] *\left[\eta_{L} / 2 k\right]\right\} \exp \left\{2 i \pi k \int_{S^{3}}\left[\eta_{L} / 2 k\right] *\left[\eta_{L} / 2 k\right]\right\} \\
\times \exp \left\{2 i \pi k \int_{S^{3}}[\alpha] *\left[\eta_{L}\right]\right\} \exp \left\{-2 i \pi \int_{S^{3}}\left[\eta_{L} / 2 k\right] *\left[\eta_{L}\right]\right\} .
\end{gathered}
$$

With the help of equation (5.3), expression (5.4) becomes

$$
\exp \left\{-(2 i \pi / 4 k) \int_{S^{3}} \eta_{L} \wedge d \eta_{L}\right\} \int d \mu_{k}([\alpha])
$$

and then

$$
\langle W(L)\rangle_{k}=\exp \left\{-(2 i \pi / 4 k) \int_{S^{3}} \eta_{L} \wedge d \eta_{L}\right\} \frac{\int d \mu_{k}([\alpha])}{\int d \mu_{k}([\alpha])} .
$$

Assuming that, for the manifold $S^{3}$, one has

$$
\int d \mu_{k}([\alpha]) \neq 0
$$

one finally obtains

$$
\begin{aligned}
\langle W(L)\rangle_{k} & =\exp \left\{-(2 i \pi / 4 k) \int_{S^{3}} \eta_{L} \wedge d \eta_{L}\right\} \\
& =\exp \left\{-(2 i \pi / 4 k) \sum_{i j} q_{i} q_{j} \int_{S^{3}} \eta_{i} \wedge d \eta_{j}\right\},
\end{aligned}
$$

which coincides with expression(5.2); and this concludes the proof.

Remark 5.1. Expression (5.2) describes an invariant of ambient isotopy (Proposition 4.2) for oriented coloured framed links. Since the matrix elements $\mathbb{L}_{i j}$ are integers, in agreement with Proposition 4.1 the observable (5.2) is invariant under the substitution $q_{i} \rightarrow q_{i}+2 k$ (for fixed $i$ ). Moreover, one can verify that Proposition 4.3 is indeed satisfied by expression (5.2).

Remark 5.2. The topological properties of knots and links in $S^{3}$ and in $\mathbb{R}^{3}$ are equal. Therefore, expression (5.2) also describes the Wilson line expectation values for the quantum Chern-Simons theory in $\mathbb{R}^{3}$ and, in fact, equation (5.2) is in agreement with the results which can be obtained by means of standard perturbation theory [33]. 


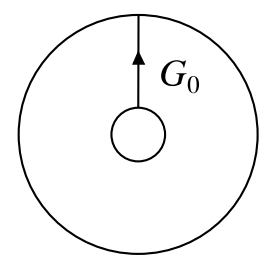

Figure 3. The region of $\mathbb{R}^{3}$ which is delimited by two spheres $S^{2}$, one into the other, with their face-toface points identified, provides a description of $S^{1} \times S^{2}$. The oriented fundamental loop $G_{0} \subset S^{1} \times S^{2}$ is also represented.

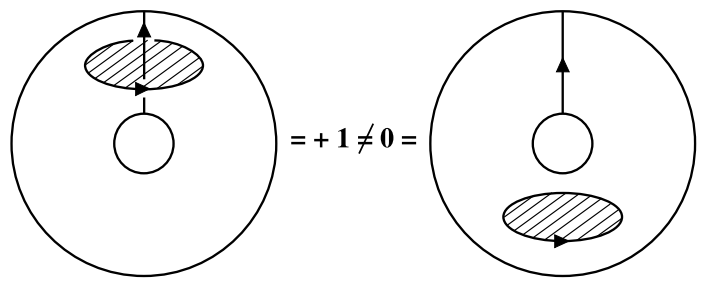

Figure 4. The trivial knot surrounding the non trivial knot $G_{0}$ is moved down (via an ambient isotopy). The intersection number of its associated surface - given by a disc - with $G_{0}$ goes from unity to 0 .

\section{Abelian Chern-Simons theory on $S^{1} \times S^{2}$}

One can represent $S^{1} \times S^{2}$ by the region of $\mathbb{R}^{3}$ which is delimited by two concentric 2 -spheres (of different radii), with the convention that the points on the two surfaces with the same angular coordinates are identified. The nontrivial knot $G_{0}$, which can be taken as generator of $H_{1}\left(S^{1} \times S^{2}, \mathbb{Z}\right) \simeq \mathbb{Z}$, is shown in Fig. 3 .

Let us recall that, since $H_{2}\left(S^{1} \times S^{2}, \mathbb{Z}\right)$ is not trivial, the linking number of two knots may not be well defined in $S^{1} \times S^{2}$; one example is shown in Fig. 4 .

Differently from $S^{3}$, the manifold $S^{1} \times S^{2}$ has nontrivial cohomology and homology groups. While $H_{D}^{3}\left(S^{1} \times S^{2}, \mathbb{Z}\right)$ is still canonically isomorphic to $\Omega^{3}\left(S^{1} \times S^{2}\right) / \Omega_{\mathbb{Z}}^{3}\left(S^{1} \times S^{2}\right)$, the group $H_{D}^{1}\left(S^{1} \times S^{2}, \mathbb{Z}\right)$ has the structure of a non trivial affine bundle over the second integral cohomology group $H^{2}\left(S^{1} \times S^{2}, \mathbb{Z}\right) \simeq \mathbb{Z}$. As shown in Fig. 1 , one can then represent $H_{D}^{1}\left(S^{1} \times S^{2}, \mathbb{Z}\right)$ by means of a collection of fibres over the base space $\mathbb{Z}$, each fibre has a linear space structure and is isomorphic to $\Omega^{1}\left(S^{1} \times S^{2}\right) / \Omega_{\mathbb{Z}}^{1}\left(S^{1} \times S^{2}\right)$. For the fiber over $0 \in \mathbb{Z}$ one can choose the trivial vanishing connection as canonical origin, so that this fibre can actually be identified with $\Omega^{1}\left(S^{1} \times S^{2}\right) / \Omega_{\mathbb{Z}}^{1}\left(S^{1} \times S^{2}\right)$. The fiber over $n \in \mathbb{Z}$, with $n \neq 0$, has not a canonical origin, but one can fix an origin and each element of this fibre will be written as a sum of this origin with an element of $\Omega^{1}\left(S^{1} \times S^{2}\right) / \Omega_{\mathbb{Z}}^{1}\left(S^{1} \times S^{2}\right)$.

\subsection{Structure of the functional measure}

The choice of an origin on each fibre of the affine bundle $H_{D}^{1}\left(S^{1} \times S^{2}, \mathbb{Z}\right)$ defines of a section $s$ of $H_{D}^{1}\left(S^{1} \times S^{2}, \mathbb{Z}\right)$ over the discrete base space $\mathbb{Z} \cong H^{2}\left(S^{1} \times S^{2}, \mathbb{Z}\right)$, with the convention that $s(0)=[0] \in H_{D}^{1}\left(S^{1} \times S^{2}, \mathbb{Z}\right)$. In agreement with property (M1), the quantum measure space $\mathcal{H}_{D}^{1}\left(S^{1} \times S^{2}, \mathbb{Z}\right)$ can also be understood as an affine bundle over $\mathbb{Z}$, and the section $s$ will be used to make the structure of the functional integral explicit. Therefore, one can actually admit distributional values for $s$ and, in fact, it is convenient to define the section $s$ with values in $\widetilde{H}_{D}^{1}\left(S^{1} \times S^{2}, \mathbb{Z}\right)$.

Definition 6.1. The simplest choice for $s$ is suggested by the additive structure of the base space. More precisely, let us pick up a nontrivial 1-cycle (or oriented knot) $G_{0}$ which is directed along 
the $S^{1}$ component of $S^{1} \times S^{2}$ and is a generator of $H_{1}\left(S^{1} \times S^{2}, \mathbb{Z}\right) \simeq \mathbb{Z}$. If $\left[\gamma_{0}\right] \in \widetilde{H}_{D}^{1}\left(S^{1} \times S^{2}, \mathbb{Z}\right)$ denotes the DB class which is canonically associated with $G_{0}$, we shall consider the section

$$
\begin{aligned}
s: & \mathbb{Z} \rightarrow \widetilde{H}_{D}^{1}\left(S^{1} \times S^{2}, \mathbb{Z}\right), \\
& n \mapsto s(n) \equiv n\left[\gamma_{0}\right] .
\end{aligned}
$$

Each element $[A]$ of $\widetilde{H}_{D}^{1}\left(S^{1} \times S^{2}, \mathbb{Z}\right)$ (and of $\left.\mathcal{H}_{D}^{1}\left(S^{1} \times S^{2}, \mathbb{Z}\right)\right)$ can then be written as

$$
[A]=n\left[\gamma_{0}\right]+[\alpha]
$$

for some integer $n$ and $[\alpha] \in \Omega^{1}\left(S^{1} \times S^{2}\right) / \Omega_{\mathbb{Z}}^{1}\left(S^{1} \times S^{2}\right)$; and the functional measure takes the form

$$
d \mu_{k}([A])=\sum_{n=-\infty}^{+\infty} D[\alpha] \exp \left\{2 i \pi k \int_{S^{1} \times S^{2}}\left(n\left[\gamma_{0}\right]+[\alpha]\right) *\left(n\left[\gamma_{0}\right]+[\alpha]\right)\right\} .
$$

Remark 6.1. Because of the translational invariance of the quantum measure, the particular choice (6.1) of the section $s$ will play no role in the computation of the observables. In fact, a modification of the origin of each fiber of $\mathcal{H}_{D}^{1}\left(S^{1} \times S^{2}, \mathbb{Z}\right)$ can be achieved by means of an element of $\Omega^{1}\left(S^{1} \times S^{2}\right) / \Omega_{\mathbb{Z}}^{1}\left(S^{1} \times S^{2}\right)$.

Expression (6.2) can be written as

$$
\begin{aligned}
d \mu_{k}([A])= & \sum_{n=-\infty}^{+\infty} D[\alpha] \exp \left\{2 i \pi k \int_{S^{1} \times S^{2}}[\alpha] *[\alpha]\right\} \exp \left\{4 i \pi k n \int_{S^{1} \times S^{2}}[\alpha] *\left[\gamma_{0}\right]\right\} \\
& \times \exp \left\{2 i \pi k n^{2} \int_{S^{1} \times S^{2}}\left[\gamma_{0}\right] *\left[\gamma_{0}\right]\right\} .
\end{aligned}
$$

As usual, in order to define $\left[\gamma_{0}\right] *\left[\gamma_{0}\right] \in \widetilde{H}_{D}^{3}\left(S^{1} \times S^{2}, \mathbb{Z}\right)$ we shall introduce a framing $G_{0 f}$ for the knot $G_{0}$ and, in agreement with equations (3.13) and (3.14), we define $\left[\gamma_{0}\right] *\left[\gamma_{0}\right] \equiv\left[\gamma_{0}\right] *\left[\gamma_{0 f}\right]=$ $[0] \in \widetilde{H}_{D}^{3}\left(S^{1} \times S^{2}, \mathbb{Z}\right)$. Therefore, with integers $k$ and $n$, the last factor entering expression (6.3) is well defined and it is equal to the identity. So, one obtains

$$
d \mu_{k}([A])=\sum_{n=-\infty}^{+\infty} D[\alpha] \exp \left\{2 i \pi k \int_{S^{1} \times S^{2}}[\alpha] *[\alpha]\right\} \exp \left\{4 i \pi k n \int_{S^{1} \times S^{2}}[\alpha] *\left[\gamma_{0}\right]\right\},
$$

with $[\alpha] \in \Omega^{1}\left(S^{1} \times S^{2}\right) / \Omega_{\mathbb{Z}}^{1}\left(S^{1} \times S^{2}\right)$.

\subsection{Zero mode}

Definition 6.2. Let $S_{0}$ be a oriented 2-dimensional sphere which is embedded in $S^{1} \times S^{2}$ in such a way that it can represent a generator of $H_{2}\left(S^{1} \times S^{2}, \mathbb{Z}\right)$.

$S_{0}$ is isotopic with the component $S^{2}$ of $S^{1} \times S^{2}$ and, if one represents $S^{1} \times S^{2}$ by the region of $\mathbb{R}^{3}$ which is delimited by two concentric spheres, $S_{0}$ can just be represented by a third concentric sphere. We shall denote by $\beta_{0}$ the distributional 1-form which is globally defined in $S^{1} \times S^{2}$ and has support on $S_{0}$; the overall sign of $\beta_{0}$ is fixed by the orientation of $S_{0}$ so that

$$
\int_{G_{0}} \beta_{0}=1
$$

Since the boundary of the closed surface $S_{0}$ is trivial, one has $d \beta_{0}=0$. For any given real parameter $x$, the 1-form $x \beta_{0}$ is also globally defined in $S^{1} \times S^{2}$; let us denote by $\left[x \beta_{0}\right] \in$ $\Omega^{1}\left(S^{1} \times S^{2}\right) / \Omega_{\mathbb{Z}}^{1}\left(S^{1} \times S^{2}\right)$ the class which is represented by the form $x \beta_{0}$. 
Proposition 6.1. For each value $m$ of the integer residues $\bmod 2 k$, the Chern-Simons measure (6.4) on $S^{1} \times S^{2}$, with nontrivial coupling constant $k$, satisfies the relation

$$
d \mu_{k}([A])=d \mu_{k}\left([A]+\left[(m / 2 k) \beta_{0}\right]\right) .
$$

Proof. From expression (6.4) one finds

$$
\begin{aligned}
d \mu_{k}\left([A]+\left[(m / 2 k) \beta_{0}\right]\right) & \\
= & \sum_{n=-\infty}^{+\infty} D[\alpha] \exp \left\{2 i \pi k \int_{S^{1} \times S^{2}}[\alpha] *[\alpha]\right\} \exp \left\{4 i \pi k n \int_{S^{1} \times S^{2}}[\alpha] *\left[\gamma_{0}\right]\right\} \\
& \times \exp \left\{4 i \pi k \int_{S^{1} \times S^{2}}[\alpha] *\left[(m / 2 k) \beta_{0}\right]\right\} \exp \left\{2 i \pi k \int_{S^{1} \times S^{2}}\left[(m / 2 k) \beta_{0}\right] *\left[(m / 2 k) \beta_{0}\right]\right\} \\
& \times \exp \left\{4 i \pi k n \int_{S^{1} \times S^{2}}\left[(m / 2 k) \gamma_{0}\right] *\left[\eta_{0}\right]\right\}
\end{aligned}
$$

where the integer $m$ takes the values $m=0,1,2, \ldots, 2 k-1$. From the equality $d \beta_{0}=0$ it follows that

$$
4 i \pi k \int_{S^{1} \times S^{2}}[\alpha] *\left[(m / 2 k) \beta_{0}\right]=2 i \pi m \int_{S^{1} \times S^{2}} \alpha \wedge d \beta_{0}=0,
$$

where $\alpha \in \Omega^{1}\left(S^{1} \times S^{2}\right)$ represents the class $[\alpha]$,

$$
2 i \pi k \int_{S^{1} \times S^{2}}\left[(m / 2 k) \beta_{0}\right] *\left[(m / 2 k) \beta_{0}\right]=i \pi\left(m^{2} / 2 k\right) \int_{S^{1} \times S^{2}} \beta_{0} \wedge d \beta_{0}=0 .
$$

Finally, relation (6.5) implies

$$
\exp \left\{4 i \pi k n \int_{S^{1} \times S^{2}}\left[(m / 2 k) \beta_{0}\right] *\left[\gamma_{0}\right]\right\}=\exp \left\{2 i \pi n m \int_{G_{0}} \beta_{0}\right\}=1 .
$$

Therefore expressions (6.7) and (6.4) are equal.

\subsection{Values of the observables}

Let us consider an oriented coloured and framed link $L$ in $S^{1} \times S^{2}$; without loss of generality, one can always assume that $L$ does not intersect the knot $G_{0}$. In agreement with equation (6.5), the integral

$$
N_{0}(L)=\int_{L} \beta_{0}
$$

takes integer values; more precisely, $N_{0}(L)$ is equal to the sum of the intersection numbers (weighted with the charges of the link components) of the link $L$ with the surface $S_{0}$.

Theorem 6.1. Given a link $L \subset S^{1} \times S^{2}$,

- when $N_{0}(L) \not \equiv 0 \bmod 2 k$, one finds $\langle W(L)\rangle_{k}=0$;

- whereas for $N_{0}(L) \equiv 0 \bmod 2 k$, one has

$$
\langle W(L)\rangle_{k}=\exp \left\{-(2 i \pi / 4 k) \int_{S^{1} \times S^{2}} \eta_{L} \wedge d \eta_{L}\right\},
$$

where $\eta_{L} \wedge d \eta_{L}$ is defined by means of the framing procedure. 
Proof. The expectation value of the Wilson line operator is given by

$$
\langle W(L)\rangle_{k}=Z_{k}^{-1} \int d \mu_{k}([A]) \exp \left\{2 i \pi \int_{S^{1} \times S^{2}}[A] *\left[\eta_{L}\right]\right\}
$$

where $d \mu_{k}([A])$ is shown in equation (6.4) and

$$
Z_{k}=\int d \mu_{k}([A])
$$

Equation (6.6) implies that $W(L)$ satisfies the following relation

$$
\begin{aligned}
\langle W(L)\rangle_{k} & =Z_{k}^{-1} \frac{1}{2 k} \sum_{m=0}^{2 k-1} \int d \mu_{k}\left([A]+\left[(m / 2 k) \beta_{0}\right]\right) e^{2 i \pi \int_{S^{1} \times S^{2}}\left([A]+\left[(m / 2 k) \beta_{0}\right]\right) *\left[\eta_{L}\right]} \\
& =Z_{k}^{-1} \int d \mu_{k}([A]) e^{2 i \pi \int_{S^{1} \times S^{2}}[A] *\left[\eta_{L}\right]} \frac{1}{2 k} \sum_{m=0}^{2 k-1} e^{2 i \pi \int_{S^{1} \times S^{2}}\left[(m / 2 k) \beta_{0}\right] *\left[\eta_{L}\right]} \\
& =\langle W(L)\rangle_{k} \frac{1}{2 k} \sum_{m=0}^{2 k-1} \exp \left\{2 i \pi(m / 2 k) \int_{L} \beta_{0}\right\} .
\end{aligned}
$$

One has

$$
\frac{1}{2 k} \sum_{m=1}^{2 k-1} \exp \left\{2 i \pi N_{0}(L) m / 2 k\right\}= \begin{cases}1 & \text { if } N_{0}(L) \equiv 0 \bmod 2 k, \\ 0 & \text { otherwise }\end{cases}
$$

Therefore equation (6.10) shows that, when $N_{0}(L) \not \equiv 0 \bmod 2 k$, the expectation value $\langle W(L)\rangle_{k}$ is vanishing.

Let us now consider the case in which $N_{0}(L) \equiv 0 \bmod 2 k$. Because of Proposition 4.1, we only need to discuss the case $N_{0}(L)=0$. In fact, if $N_{0}(L)=2 k p$ for some integer $p \neq 0$, at least one of the link components $C \subset L$ intersects $S_{0}$; one can then modify the value $q_{C}$ of its charge according to $q_{C} \rightarrow q_{C}-2 k p$ so that $N_{0}(L)$ vanishes. According to the decomposition $[A]=n\left[\gamma_{0}\right]+[\alpha]$, one finds

$$
\begin{aligned}
\exp \left\{2 i \pi \int_{S^{1} \times S^{2}}[A] *\left[\eta_{L}\right]\right\} & =\exp \left\{2 i \pi n \int_{S^{1} \times S^{2}}\left[\gamma_{0}\right] *\left[\eta_{L}\right]\right\} \exp \left\{2 i \pi \int_{S^{1} \times S^{2}}[\alpha] *\left[\eta_{L}\right]\right\} \\
& =\exp \left\{2 i \pi \int_{S^{1} \times S^{2}}[\alpha] *\left[\eta_{L}\right]\right\},
\end{aligned}
$$

where the last equality is a consequence of the identity $\left[\gamma_{0}\right] *\left[\eta_{L}\right]=[0] \in \widetilde{H}_{D}^{3}\left(S^{1} \times S^{2}, \mathbb{Z}\right)$, which follows from the framing procedure. Then, from equation (6.9) one gets

$$
\langle W(L)\rangle_{k}=Z_{k}^{-1} \int \sum_{n=-\infty}^{+\infty} D[\alpha] e^{2 i \pi k \int_{S^{1} \times S^{2}}[\alpha] *[\alpha]} e^{4 i \pi k n \int_{S^{1} \times S^{2}}[\alpha] *\left[\gamma_{0}\right]} e^{2 i \pi \int_{S^{1} \times S^{2}}[\alpha] *\left[\eta_{L}\right]} .
$$

When $N_{0}(L)=0$, the link $L$ is homological trivial and one can find a Seifert surface for $L$. More precisely, in agreement with Proposition 4.3 and equation (4.15), one can substitute $L$ with its simplicial satellite $\bar{L}$, defined in Section 4, whose components have unitary charges. The oriented framed link $\bar{L} \subset S^{1} \times S^{2}$ also is homologically trivial and it is the boundary of an oriented surface that we shall denote by $\Sigma_{\bar{L}} \subset S^{1} \times S^{2}$. Let $\eta_{L}$ be the distributional 1-form with support on $\Sigma_{\bar{L}}$ which is globally defined in $S^{1} \times S^{2}$; because of Proposition 4.3, in the Chechde Rham description of the DB classes, $\left[\eta_{L}\right]$ can then be represented by the sequence $\left(\eta_{L}, 0,0\right)$. 


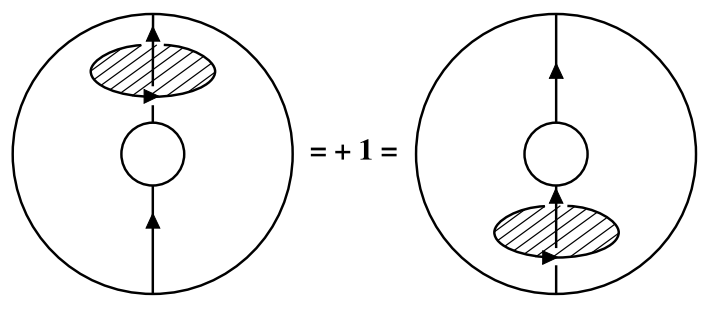

Figure 5. An example of conservation of the intersection number under ambient isotopy for a globally trivial 1-cycle.

The 1 -form $(1 / 2 k) \eta_{L}$ also is globally defined in $S^{1} \times S^{2}$ and we shall denote by $\left[(1 / 2 k) \eta_{L}\right]$ the DB class which is represented by the form $(1 / 2 k) \eta_{L}$. By construction,

$$
\exp \left\{-4 i \pi k \int_{S^{1} \times S^{2}}[\alpha] *\left[(1 / 2 k) \eta_{L}\right]\right\}=\exp \left\{-2 i \pi \int_{S^{1} \times S^{2}}[\alpha] *\left[\eta_{L}\right]\right\}
$$

and the condition $N_{0}(L)=0\left(\right.$ or $\left.N_{0}(L) \equiv 0 \bmod 2 k\right)$ implies that, for integer $n$,

$$
\exp \left\{-4 i \pi k n \int_{S^{1} \times S^{2}}\left[(1 / 2 k) \eta_{L}\right] *\left[\gamma_{0}\right]\right\}=1
$$

By means of the substitution $[\alpha] \rightarrow[\alpha]-\left[(1 / 2 k) \eta_{L}\right]$ and with the help of equations $(6.12)$ and (6.13), expression (6.11) assumes the form

$$
\langle W(L)\rangle_{k}=\exp \left\{-(2 i \pi / 4 k) \int_{S^{1} \times S^{2}} \eta_{L} \wedge d \eta_{L}\right\} Z_{k}^{-1} Z_{k}
$$

Therefore, assuming $Z_{k} \neq 0$, when $N_{0}(L) \equiv 0 \bmod 2 k$ one gets

$$
\langle W(L)\rangle_{k}=\exp \left\{-(2 i \pi / 4 k) \int_{S^{1} \times S^{2}} \eta_{L} \wedge d \eta_{L}\right\}
$$

and this concludes the proof.

Remark 6.2. Expression (6.8) formally coincides with the result (5.5) which has been obtained in the case $M_{3} \sim S^{3}$. It should be noted that the integral (which appears in equation (6.8))

$$
\int_{S^{1} \times S^{2}} \eta_{L} \wedge d \eta_{L} \equiv \int_{S^{1} \times S^{2}} \eta_{L} \wedge d \eta_{\bar{L}_{f}}=\int_{\bar{L}_{f}} \beta_{L}
$$

where $\bar{L}_{f}$ denotes the framing of $\bar{L}$, is well defined because it does not depend on the choice of the Seifert surface of $\bar{L}$. Indeed suppose that, instead of $\Sigma_{\bar{L}}$, we take $\Sigma_{\bar{L}}^{\prime}$ as Seifert surface for the link $\bar{L}$. The difference between the intersection number (6.14) of $\bar{L}_{f}$ with $\Sigma_{\bar{L}}^{\prime}$ and $\Sigma_{\bar{L}}$ is given by the intersection number of $\bar{L}_{f}$ with the closed surface $\Sigma_{\bar{L}}^{\prime} \cup \Sigma_{\bar{L}}^{-1}$. This surface could be nontrivial in $S^{1} \times S^{2}$ but, since $\bar{L}$ is homologically trivial, $\bar{L}_{f}$ also is homologically trivial and then its intersection number with a closed surface vanishes. The example of Fig. 5 illustrates the ambient isotopy invariance of the intersection number of a homologically trivial link with the Seifert surface of a trivial knot in $S^{1} \times S^{2}$. 


\section{Abelian Chern-Simons theory on $S^{1} \times \Sigma_{g}$}

Let us now consider the manifold $M_{3} \sim S^{1} \times \Sigma_{g}$ where $\Sigma_{g}$ is a closed Riemann surface of genus $g \geq 1$. In this case, the computation of the Chern-Simons observables is rather similar to the computation when $M_{3} \sim S^{1} \times S^{2}$. So, we shall briefly illustrate the main steps of the construction.

As it has been mentioned in Section $1, H_{D}^{1}\left(S^{1} \times \Sigma_{g}, \mathbb{Z}\right)$ has the structure of a affine bundle over $H^{2}\left(S^{1} \times \Sigma_{g}, \mathbb{Z}\right) \sim \mathbb{Z}^{2 g+1}$ with $\Omega^{1}\left(S^{1} \times \Sigma_{g}\right) / \Omega_{\mathbb{Z}}^{1}\left(S^{1} \times \Sigma_{g}\right)$ acting canonically on each fibre by translation. In agreement with property $(\mathbf{M} 1)$, the functional space $\mathcal{H}_{D}^{1}\left(S^{1} \times \Sigma_{g}, \mathbb{Z}\right)$ is assumed to have the same structure of $H_{D}^{1}\left(S^{1} \times \Sigma_{g}, \mathbb{Z}\right)$ and, in order to fix a origin in each fibre, we need to introduce a section $s: \mathbb{Z}^{2 g+1} \rightarrow \mathcal{H}_{D}^{1}\left(S^{1} \times \Sigma_{g}, \mathbb{Z}\right)$.

Definition 7.1. Let the nonintersecting oriented framed knots $\left\{G_{0}, G_{1}, \ldots, G_{2 g}\right\}$ in $S^{1} \times \Sigma_{g}$ represent the generators of $H_{1}\left(S^{1} \times \Sigma_{g}, \mathbb{Z}\right)$. For each $j=0,1, \ldots, 2 g$, we shall denote by $\left[\gamma_{j}\right] \in \widetilde{H}_{D}^{1}\left(S^{1} \times \Sigma_{g}, \mathbb{Z}\right)$ the DB class which is canonically associated with the knot $G_{j}$.

Definition 7.2. If the elements of $\mathbb{Z}^{2 g+1}$ are represented by vectors

$$
\vec{n} \equiv\left(n_{0}, n_{1}, n_{2}, \ldots, n_{2 g}\right) \in \mathbb{Z}^{2 g+1},
$$

a possible choice for the section $s$ is given by

$$
\begin{aligned}
s: & \mathbb{Z}^{2 g+1} \rightarrow \widetilde{H}_{D}^{1}\left(S^{1} \times \Sigma_{g}, \mathbb{Z}\right), \\
& \vec{n} \mapsto s(\vec{n})=[n \gamma] \equiv \vec{n} \cdot[\vec{\gamma}]=\sum_{j=0}^{2 g} n_{j}\left[\gamma_{j}\right] .
\end{aligned}
$$

Each class $[A] \in \widetilde{H}_{D}^{1}\left(S^{1} \times \Sigma_{g}, \mathbb{Z}\right)$ can then be written as

$$
[A]=[n \gamma]+[\alpha]
$$

for certain $\vec{n}$ and $[\alpha] \in \Omega^{1}\left(S^{1} \times \Sigma_{g}\right) / \Omega_{\mathbb{Z}}^{1}\left(S^{1} \times \Sigma_{g}\right)$. Consequently, the Chern-Simons functional measure takes the form

$$
d \mu_{k}([A])=\sum_{\vec{n}} D[\alpha] \exp \left\{2 i \pi k \int_{S^{1} \times S^{2}}[\alpha] *[\alpha]\right\} \exp \left\{4 i \pi k \int_{S^{1} \times S^{2}}[\alpha] *[n \gamma]\right\},
$$

which is the analogue of equation (6.4). The condition $[n \gamma] *[n \gamma]=0 \in \widetilde{H}_{D}^{3}\left(S^{1} \times \Sigma_{g}, \mathbb{Z}\right)$, which results from the framing procedure, has already been used to simplify the expression of $d \mu_{k}([A])$.

Definition 7.3. Let the oriented closed surfaces $S_{j} \subset S^{1} \times \Sigma_{g}$, with $j=0,1, \ldots, 2 g$, represent the generators of $H_{2}\left(S^{1} \times \Sigma_{g}, \mathbb{Z}\right) \sim \mathbb{Z}^{2 g+1}$. We shall denote by $\beta_{j} \in \widetilde{H}_{D}^{1}\left(S^{1} \times \Sigma_{g}, \mathbb{Z}\right)$ the distributional 1-form which is globally defined in $S^{1} \times \Sigma_{g}$ and has support on $S_{j}$. One can choose the generators of $H_{2}\left(S^{1} \times \Sigma_{g}, \mathbb{Z}\right)$ in such a way that the following orthogonality relations are satisfied

$$
\int_{G_{i}} \beta_{j}=\delta_{i j}, \quad i, j=0,1, \ldots, 2 g .
$$

Since $S_{j}$ are closed surfaces, one has $d \beta_{j}=0$. For any real parameter $x$, the 1 -form $x \beta_{j}$ also is globally defined in $S^{1} \times \Sigma_{g}$ and the corresponding class, which can be represented by $x \beta_{j}$, will be denoted by $\left[x \beta_{j}\right] \in \Omega^{1}\left(S^{1} \times \Sigma_{g}\right) / \Omega_{\mathbb{Z}}^{1}\left(S^{1} \times \Sigma_{g}\right)$. The arguments that have been presented to prove Proposition 6.1 can also be used to prove the following 
Proposition 7.1. The quantum measure (7.1) of the Chern-Simons theory on $S^{1} \times \Sigma_{g}$, with nontrivial coupling constant $k$, satisfies the relation

$$
d \mu_{k}([A])=d \mu_{k}\left([A]+\left[(m / 2 k) \beta_{j}\right]\right) .
$$

for $m=0,1,2, \ldots, 2 k-1$ and for each value of $j=0,1, \ldots, 2 g$.

Finally, the expectation values of the Wilson line operators are determined by the following

Theorem 7.1. Let $L$ be a oriented coloured framed link in $S^{1} \times \Sigma_{g}$. For each $j=0,1, \ldots, 2 g$, let us introduce the integer

$$
N_{j}(L)=\int_{L} \beta_{j}
$$

Then

- when $N_{j}(L) \not \equiv 0 \bmod 2 k$ for at least one value of $j=0,1, \ldots, 2 g$, one has $\langle W(L)\rangle_{k}=0$;

- whereas when $N_{j}(L) \equiv 0 \bmod 2 k$ for all values of $j=0,1, \ldots, 2 g$, one finds

$$
\langle W(L)\rangle_{k}=\exp \left\{-(2 i \pi / 4 k) \int_{S^{1} \times \Sigma_{g}} \eta_{L} \wedge d \eta_{L}\right\},
$$

where $\eta_{L} \wedge d \eta_{L}$ is defined by means of the framing procedure.

Proof. The proof is similar to the proof of Theorem 6.1. In fact, when $N_{j}(L) \not \equiv 0 \bmod 2 k$ for at least one value of $j=0,1, \ldots, 2 g$, Proposition 7.1 implies that the Chern-Simons expectation value $\langle W(L)\rangle_{k}$ vanishes. On the other hand, when $N_{j}(L) \equiv 0 \bmod 2 k$ for all values of $j=$ $0,1, \ldots, 2 g$, the substitution $[\alpha] \rightarrow[\alpha]-\left[(1 / 2 k) \eta_{L}\right]$ in the functional measure (7.1) leads to the equation (7.2). It should be noted that expression (7.2) is well defined because the link $L$ and then its framing $L_{f}$ are homologically trivial.

\section{Surgery rules}

For the quantum Abelian Chern-Simons theory on the manifolds $S^{1} \times S^{2}$ and $S^{1} \times \Sigma_{g}$ (and, in general, in any nontrivial 3-manifold), the standard gauge theory approach which is based on the gauge group $U(1)$ is in principle well defined but presents some technical difficulties, which are related, for instance, to the implementation of the gauge fixing procedure and the determination of the Feynman propagator. As a matter of facts, by means of the usual methods of quantum gauge theories, the computation of the Chern-Simons observables in a nontrivial 3-manifold has never been explicitly produced.

In order to determine the Wilson line expectation values in $M_{3} \nsim S^{3}$, one can use for instance the surgery rules of the Reshetikhin-Turaev type [6] as developed by Lickorish [39] and by Morton and Strickland [40]. In this section, we outline the surgery method which turns out to produce the Chern-Simons observables for the manifolds $S^{1} \times S^{2}$ and $S^{1} \times \Sigma_{g}$ in complete agreement with the results obtained in the DB approach of the path-integral.

Every closed orientable connected 3-manifold $M_{3}$ can be obtained by Dehn surgery on $S^{3}$ and admits a surgery presentation [29] which is described by a framed surgery link $\mathcal{L} \subset S^{3}$ with integer surgery coefficients. Each surgery coefficient specifies the framing of the corresponding component of $\mathcal{L}$ because it coincides with the linking number of this component with its framing. The manifold $S^{1} \times S^{2}$ admits a presentation with surgery link given by the unknot with vanishing surgery coefficient, whereas $S^{1} \times S^{1} \times S^{1}$ for example corresponds to the Borromean rings with vanishing surgery coefficients. Any oriented coloured framed link $L \subset M_{3}$ can be described by a link $L^{\prime}=L \cup \mathcal{L}$ in $S^{3}$ in which: 
- the surgery link $\mathcal{L}$ describes the surgery instructions corresponding to a presentation of $M_{3}$ in terms of Dehn surgery on $S^{3}$;

- the remaining components of $L^{\prime}$ describe how $L$ is placed in $M_{3}$.

Assuming that the expectation values of the Wilson line operators form a complete set of observables, one can find [33] consistent surgery rules, according to which the expectation value of the Wilson line operator $W(L)$ in $M_{3}$ can be written as a ratio

$$
\left.\langle W(L)\rangle_{k}\right|_{M_{3}}=\left.\langle W(L) W(\mathcal{L})\rangle_{k}\right|_{S^{3}} /\left.\langle W(\mathcal{L})\rangle_{k}\right|_{S^{3}},
$$

where to each component of the surgery link is associated a particular colour state $\psi_{0}$. Remember that, for fixed integer $k$, the colour space coincides with space of residue classes of integers mod $2 k$, which has a canonical ring structure; let $\chi_{j}$ denote the residue class associated with the integer $j$. Then, the colour state $\psi_{0}$ is given by

$$
\psi_{0}=\sum_{j=0}^{2 k-1} \chi_{j} .
$$

One can verify that the surgery rule (8.1) is well defined and consistent; in fact, expression (8.1) is invariant under Kirby moves [41]. Finally, one can check that, according to the surgery formula (8.1), the expectation values of the Wilson line operators in $S^{1} \times S^{2}$ and in $S^{1} \times \Sigma_{g}$ are given precisely by the expressions of Theorems 6.1 and 7.1, which have been obtained by means of the DB cohomology.

\section{Conclusions}

In the standard field theory formulation of Abelian gauge theories, the (classical fields) configuration space is taken to be the set of 1 -forms modulo closed forms. But when the observables of the theory are given by the exponential of the holonomies which are associated with oriented loops, the classical configuration space is actually given by the set of 1-forms modulo forms of integer periods; that is, the classical configuration space indeed coincides with space of the Deligne-Beilinson cohomology classes. So, in this article we have considered the Abelian ChernSimons gauge theory, in which a complete set of observables is given by the set of exponentials of the holonomies which are associated with oriented knots or links in a 3-manifold $M_{3}$. We have explored the main properties of the quantum theory and of the corresponding quantum functional integral, which enters the computation of the observables, when the path-integral is really defined over the Deligne-Beilinson classes. Within this new approach, we have produced an explicit path-integral computation of the Chern-Simons link invariants in a class of torsionfree 3-manifolds. In facts, we have not used any standard gauge-fixing and perturbative method, as it has been done so far in literature. Our results are based on an explicit non-perturbative path-integral computation and are exact results.

Let us briefly summarize the main issues of our article. In Sections 2 and 3 we have discussed a few technical points which are important for the computation of the observables. The basic definitions and properties of the DB cohomology together with a distributional extension of the space of the equivalence classes have been illustrated. Then we have shown how the framing procedure, which is used to give a topological meaning to the self-linking number, can be naturally defined also in the DB context. The general features of the Abelian Chern-Simons theory in a generic 3-manifold $M_{3}$ have been derived in Section 4. The main achievements concerning the observables are the "colour periodicity" property (Proposition 4.1), the "ambient isotopy invariance" (Proposition 4.2) and the validity of appropriate "satellite relations" (Proposition 4.3). 
With respect to the standard field theory approach, our proofs extend the validity of these properties from $\mathbb{R}^{3}$ to a generic (closed and oriented) manifold $M_{3}$.

The Abelian Chern-Simons theory formulated in $S^{3}$ is discussed in Section 5 and its solution is given by Theorem 5.1; in this case, the outcome is in agreement with the results obtained by means of standard perturbation theory in $\mathbb{R}^{3}$. The expressions of the observables for the Chern-Simons theory formulated in $S^{1} \times S^{2}$ and in a generic 3-manifold of the type $S^{1} \times \Sigma_{g}$ are contained in Theorems 6.1 and 7.1; in the standard field theory approach, no proof of these theorems actually exists.

Finally, we have checked the validity our path-integral results by means of an alternative "combinatorial method". Indeed, the link invariants defined in the Chern-Simons theory are related to the link invariants defined by means of the quantum group methods of Reshetikhin and Turaev. Given a surgery presentation in $S^{3}$ of a generic 3-manifold $M_{3}$ and knowing the values of the link invariants in $S^{3}$, one can use the surgery method of Lickorish and Morton-Strickland to determine the values of the link invariants in $M_{3}$. As far as the Abelian Chern-Simons is concerned, we have presented the basic aspects of this surgery method in Section 8. We have verified that the expression of the link invariants for the manifolds $S^{1} \times S^{2}$ and $S^{1} \times \Sigma_{g}$, which are described by Theorems 6.1 and 7.1, precisely coincide with the results obtained by means of the surgery method.

Clearly, in the case of a generic 3-manifold, the general features of the Deligne-Beilinson approach to the Abelian Chern-Simons functional integral remain to be fully explored. Possible applications of this formalism to the non-Abelian Chern-Simons theory would also give new hints on the topological meaning of the polynomial link invariants. Finally, we mention that extensions of Deligne-Beilinson cohomology approach to the topological field theories in lower dimensions can easily be produced, but the resulting structure of the observables appears to be quite elementary. Presumably, applications in higher dimensions will produce more interesting invariants.

\section{Acknowledgements}

We wish to thank Raymond Stora for useful discussions.

\section{References}

[1] Schwarz A.S., The partition function of degenerate quadratic functional and Ray-Singer invariants, Lett. Math. Phys. 2 (1978), 247-252.

Schwarz A.S., The partition function of a degenerate functional, Comm. Math. Phys. 67 (1979), 1-16.

[2] Hagen C.R., A new gauge theory without an elementary photon, Ann. Physics 157 (1984), 342-359.

[3] Polyakov A.M., Fermi-Bose transmutations induced by gauge fields, Modern Phys. Lett. A 3 (1988), 325328.

[4] Witten E., Quantum field theory and the Jones polynomial, Comm. Math. Phys. 121 (1989), 351-399.

[5] Jones V.F.R., A polynomial invariant for knots via von Neumann algebras, Bull. Amer. Math. Soc. (N.S.) 12 (1985), 103-111.

Jones V.F.R., Hecke algebra representations of braid groups and link polynomials, Ann. of Math. (2) 126 (1987), 335-388.

[6] Reshetikhin N.Y., Turaev V.G., Ribbon graphs and their invariants derived from quantum groups, Comm. Math. Phys. 127 (1990), 1-26.

Reshetikhin N.Y., Turaev V.G., Invariants of 3-manifolds via link polynomials and quantum groups, Invent. Math. 103 (1991), 547-597.

[7] Deligne P., Théorie de Hodge. II, Inst. Hautes Études Sci. Publ. Math. (1971), no. 40, 5-58.

[8] Beilinson A.A., Higher regulators and values of L-functions, J. Soviet Math. 30 (1985), 2036-2070. 
[9] Esnault H., Viehweg E., Deligne-Beilinson cohomology, in Beilinson's Conjectures on Special Values of $L$ Functions, Editors M. Rapaport, P. Schneider and N. Schappacher, Perspect. Math., Vol. 4, Academic Press, Boston, MA, 1988, 43-91.

[10] Jannsen U., Deligne homology, Hodge- $D$-conjecture, and motives, in Beilinson's Conjectures on Special Values of L-Functions, Editors M. Rapaport, P. Schneider and N. Schappacher, Perspect. Math., Vol. 4, Academic Press, Boston, MA, 1988, 305-372.

[11] Brylinski J.L., Loop spaces, characteristic classes and geometric quantization, Progress in Mathematics, Vol. 107, Birkhäuser Boston, Inc., Boston, MA, 1993.

[12] Cheeger J., Simons J., Differential characters and geometric invariants, Stony Brook Preprint, 1973 (reprinted in Geometry and Topology Proc. (1983-84), Editors J. Alexander and J. Harer, Lecture Notes in Math., Vol. 1167, Springer, Berlin, 1985, 50-90).

[13] Koszul J.L., Travaux de S.S. Chern et J. Simons sur les classes caractéristiques, Seminaire Bourbaki, Vol. 1973/1974, Lecture Notes in Math., Vol. 431, Springer, Berlin, 1975, 69-88.

[14] Harvey R., Lawson B., Zweck J., The de Rham-Federer theory of differential characters and character duality, Amer. J. Math. 125 (2003), 791-847, math.DG/0512251.

[15] Alvarez M., Olive D.I., The Dirac quantization condition for fluxes on four-manifolds, Comm. Math. Phys. 210 (2000), 13-28, hep-th/9906093.

Alvarez M., Olive D.I., Spin and Abelian electromagnetic duality on four-manifolds, Comm. Math. Phys. 217 (2001), 331-356, hep-th/0003155.

[16] Alvarez A., Olive D.I., Charges and fluxes in Maxwell theory on compact manifolds with boundary, Comm. Math. Phys. 267 (2006), 279-305, hep-th/0303229.

[17] Alvarez O., Topological quantization and cohomology, Comm. Math. Phys. 100 (1985), 279-309.

[18] Gawedzki K., Topological Actions in two-dimensional quantum field theories, in Nonperturbative Quantum Field Theory (Cargèse, 1987), NATO Adv. Sci. Inst. Ser. B Phys., Vol. 185, Plenum, New York, 1988, 101-141.

[19] Witten E., Topological quantum field theory, Comm. Math. Phys. 117 (1988), 353-386.

[20] Witten E., Dynamics of quantum field theory, in Quantum Fields and Strings: A Course for Mathematicians (Princeton, NJ, 1996/1997), Editors P. Deligne et al., Amer. Math. Soc., Providence, RI, 1999, Vol. 2, 1119 1424.

[21] Freed D.S., Locality and integration in topological field theory, in Group Theoretical Methods in Physics, Vol. 2, Editors M.A. del Olmo, M. Santander and J.M. Guilarte, CIEMAT, 1993, 35-54, hep-th/9209048.

[22] Zucchini R., Relative topological integrals and relative Cheeger-Simons differential characters J. Geom. Phys. 46 (2003), 355-393, hep-th/0010110.

Zucchini R., Abelian duality and Abelian Wilson loops, Comm. Math. Phys. 242 (2003), 473-500, hep-th/0210244.

[23] Hopkins M.J., Singer I.M., Quadratic functions in geometry, topology, and M-theory, J. Differential Geom. 70 (2005), 329-452, math.AT/0211216.

[24] Woodhouse N.M.J., Geometric quantization, 2nd ed., Oxford Mathematical Monographs, Oxford Science Publications, The Clarendon Press, Oxford University Press, New York, 1992.

[25] Bauer M., Girardi G., Stora R., Thuillier F., A class of topological actions, J. High Energy Phys. 2005 (2005), no. 8, 027, 35 pages, hep-th/0406221.

[26] Mackaay M., Picken R., Holonomy and parallel transport for Abelian gerbes, Adv. Math. 170 (2002), 287339, math.DG/0007053.

[27] Godement R., Topologie algébrique et théorie des faisceaux, Actualit'es Sci. Ind., no. 1252, Publ. Math. Univ. Strasbourg, no. 13, Hermann, Paris, 1958 (reprinted, 1998).

[28] Bott R., Tu L.W., Differential forms in algebraic topology, Graduate Texts in Mathematics, Vol. 82, SpringerVerlag, New York - Berlin, 1982.

[29] Rolfsen D., Knots and links, Mathematics Lecture Series, no. 7, Publish or Perish, Inc., Berkeley, Calif., 1976.

[30] Calugareanu G., Sur les classes d'isotopie des noeuds tridimensionnels et leurs invariants, Czechoslovak Math. J. 11 (1961), 588-625.

[31] Calugareanu G., L'intégrale de Gauss et l'Analyse des noeuds tridimensionnels, Rev. Math. Pures Appl. 4 (1959), 5-20.

Pohl W.F., The self-linking number of a closed space curve, J. Math. Mech. 17 (1967/1968), 975-985. 
[32] Guadagnini E., Martellini M., Mintchev M., Wilson lines in Chern-Simons theory and link invariants, Nuclear Phys. B 330 (1990), 575-607.

[33] Guadagnini E., The link invariants of the Chern-Simons field theory. New developments in topological quantum field theory, de Gruyter Expositions in Mathematics, Vol. 10, Walter de Gruyter \& Co., Berlin, 1993.

[34] Feynman R.P., Hibbs A.R., Quantum mechanics and path integrals, McGraw-Hill, New York, 1965.

[35] Coleman S., Aspects of symmetry, Cambridge University Press, New York, 1985.

[36] Elworthy D. and Truman A., Feynman maps, Cameron-Martin formulae and anharmonic oscillators, Ann. Inst. Henri Poincaré Phys. Théor. 41 (1984), 115-142.

[37] Ashtekar A., Lewandowski J., Representation theory of analytic holonomy $C^{*}$-algebras, in Knots and Quantum Gravity (Riverside, CA, 1993), Editors J. Baez, Oxford Lecture Ser. Math. Appl., Vol. 1, Oxford Univ. Press, New York, 21-61, gr-qc/9311010.

[38] Baez J.C., Link invariants, holonomy algebras, and functional integration, J. Funct. Anal. 127 (1995), 108-131, hep-th/9301063.

[39] Lickorish W.B.R., Invariants for 3-manifolds from the combinatorics of the Jones polynomial, Pacific J. Math. 149 (1991), 337-386.

[40] Morton H.R., Strickland P.M., Satellites and surgery invariants, in Knots 90 (Osaka, 1990), Editor A. Kawauchi, de Gruyter, Berlin, 1992.

[41] Kirby R., A calculus for framed links in $S^{3}$, Invent. Math. 45 (1978), 35-56. 